\title{
A New Study on Halpern and Nonconvex Combination Algorithm for Nonlinear Mappings in Banach Spaces with Applications
}

\author{
Gaobo Li (iD) \\ School of Education, Shandong Women's University, Jinan 250300, China \\ Correspondence should be addressed to Gaobo Li; gaobolisdwu@163.com
}

Received 29 November 2020; Accepted 30 December 2020; Published 29 January 2021

Academic Editor: Sun Young Cho

Copyright (c) 2021 Gaobo Li. This is an open access article distributed under the Creative Commons Attribution License, which permits unrestricted use, distribution, and reproduction in any medium, provided the original work is properly cited.

\begin{abstract}
In this paper, we introduce a Halpern algorithm and a nonconvex combination algorithm to approximate a solution of the split common fixed problem of quasi- $\phi$-nonexpansive mappings in Banach space. In our algorithms, the norm of linear bounded operator does not need to be known in advance. As the application, we solve a split equilibrium problem in Banach space. Finally, some numerical examples are given to illustrate the main results in this paper and compare the computed results with other ones in the literature. Our results extend and improve some recent ones in the literature.
\end{abstract}

\section{Introduction}

Let $H_{1}$ be a Hilbert space, and let $C$ be the nonempty closed convex subset of $H_{1}$. Let $H_{2}$ be a real Hilbert space, and let $Q$ be the nonempty closed convex subset of $\mathrm{H}_{2}$. Let A: $H_{1} \longrightarrow H_{2}$ be a linear bounded operator. In 1994, Censor and Elfving [1] introduced the split feasibility problem (SFP) as a generalization of convex feasibility problem as follows:

$$
\text { find a point } x^{*} \in C \text { such that } A x^{*} \in Q \text {. }
$$

Recntly, the SFP and its variants have been investigated by many authors due to its real applications such as medical imaging, radiation therapy, and treatment planning; see, e.g., [2-5]. For solving SFP (1), it needs to get the inverse $A^{-1}$ (assuming the existence of $A^{-1}$ ) in algorithm of Censor and Elfving [1]. However, few authors continue to study the algorithm of Censor and Elfving since the difficulty of computing $A^{-1}$, even if it exists. In fact, another algorithm solving SFP (1) is more popular which is called CQ algorithm given by Byrne $[6,7]$. The CQ algorithm of Byrne is a gradient projection method in convex minimization. Since the CQ algorithm does need to compute $A^{-1}$ and only involves the projections $P_{C}$ and $P_{Q}$, it is easy to implement when $P_{C}$ and $P_{Q}$ have the closed-form expressions. However, the computations of $P_{C}$ and $P_{Q}$ are also difficult if these projections did not have the closed-form expressions which is such that the CQ algorithm of Byrne $[6,7]$ is not easy to implement in this case. In 2010, Xu [8] investigated the CQ algorithm from the ways of optimization and fixed point, proposed Mann's algorithm, and relaxed CQ algorithm to solve SFP (1). In the relaxed CQ algorithm, the sets C and $Q$ are level sets of convex functions so that the projections involved in the CQ algorithm are onto half-spaces, which makes the algorithm implementable. Also, in 2010, Moudafi [9] proposed an iterative method to solve a split common fixed point problem for quasi-nonexpansive mappings in which the projection is not involved which is such that the algorithm is easy to implement. In 2014, Kraikaew and Saejung [10] combined the Moudafi method and the Halpern algorithm to propose a new iteration in which the projection is not involved for solving the SFP. In the recent years, many algorithms have been given to solve the SFP in Hilbert spaces; see, for instance, [11-15] and the references therein.

However, because of the complexity of properties in Banach space, it is very difficulty to solve SFP (and fixed point problem) in Banach spaces. Until now, only limited 
works on SFP (and fixed point problem) in Banach spaces have been reported in the literature. For instance, the authors in [16] gave an algorithm to solve SFP in Banach space. In [17], Tang et al. introduced some iterative algorithms to solve a split common fixed point problem for a quasi-strict pseudocontractive mapping and an asymptotically nonexpansive mapping in two Banach spaces and obtained the weak and strong convergence for the proposed algorithms. In [18], Chen et al. proposed a new hybrid projection method for solving split feasibility and fixed point problems involved in Bregman quasi-strictly pseudocontractive mapping in $p$-uniformly convex and uniformly smooth real Banach spaces. They proved the strong convergence for the proposed algorithm using the Bregman projection method. On the feasible and common fixed point problem, the authors also refer to [19-21].

Let $E_{1}$ be a 2-uniformly convex and 2-uniformly smooth real Banach space with the best smoothness constant $k>0$ and $E_{2}$ be a uniformly smooth, strictly convex, and reflective Banach space. Let $S: E_{1} \longrightarrow E_{1}$ be a closed quasi- $\phi$-nonexpansive mapping and $A: E_{1} \longrightarrow E_{2}$ be a linear bounded operator. Very recently, $\mathrm{Ma}$ et al. [22] proposed a hybrid projection algorithm to solve the following split feasibility problem and fixed point problem:

$$
\text { find } x^{*} \in C \text { such that } A x^{*} \in Q \text {, }
$$

where $C=\left\{x \in E_{1}: x=S x\right\}$ and $Q \subset E_{2}$ is a nonempty closed convex subset. Precisely, their algorithm to solve (2) is as follows:

$$
\left\{\begin{array}{l}
x_{1} \in E_{1}, C_{1}=E_{1}, \\
z_{n}=J^{-1}\left(J_{1} x_{n}+\gamma A^{*} J_{2}\left(P_{Q}-I\right) A x_{n}\right), \\
y_{n}=J^{-1}\left[\alpha_{n} J_{1} z_{n}+\left(1-\alpha_{n}\right) J_{1} S z_{n}\right], \\
C_{n+1}=\left\{v \in C_{n}: \phi\left(v, y_{n}\right) \leq \phi\left(v, x_{n}\right), \phi\left(v, z_{n}\right) \leq \phi\left(v, x_{n}\right)\right\}, \\
x_{n+1}=\Pi_{C_{n+1}} x_{1}, \quad n \geq 1,
\end{array}\right.
$$

where $\{\alpha\} \subset[\delta, 1)$ with $\delta>0, \gamma \in\left(0,\left(1 /\|A\|^{2} k^{2}\right)\right), P_{Q}$ is the metric projection of $E_{2}$ onto $Q$, and $\Pi_{C_{n+1}}$ is the generalized projection of $E_{1}$ in $C_{n+1}$. The authors proved that the sequence generated by (3) strongly converges to a point which solves (2).

On the contrary, the most algorithms of approximating the fixed points of quasi- $\phi$-nonexpansive mappings in Banach spaces are constructed by the hybrid or shrinking projection methods, see [23-25]. However, in 2018, Hieu and Strodiot [26] introduced a new iterative algorithm for solving pseudomonotone equilibrium problem involving the fixed point problem for quasi- $\phi$-nonexpansive mapping in Banach space without using the hybrid or shrinking projection methods. More precisely, their algorithm is

$$
\left\{\begin{array}{l}
y_{n}=\operatorname{argmin}\left\{\lambda_{n} f\left(x_{n}, y\right)+\frac{1}{2} \phi\left(y, x_{n}\right): y \in C\right\}, \\
z_{n}=\operatorname{argmin}\left\{\lambda_{n} f\left(y_{n}, y\right)+\frac{1}{2} \phi\left(y, x_{n}\right): y \in C\right\}, \\
x_{n+1}=\Pi_{C}\left(J^{-1}\left(\alpha_{n} J u+\left(1-\alpha_{n}\right)\left(\beta_{n} J z_{n}+\left(1-\beta_{n}\right) J S z_{n}\right)\right)\right),
\end{array}\right.
$$

where $f: C \times C \longrightarrow \mathbb{R}$ is a pseudomonotone bifunction and $S: C \longrightarrow C$ is a quasi- $\phi$-nonexpansive mapping. The authors proved that the sequence generated by (4) strongly converges to a common point that solves the pseudomonotone equilibrium problem on $f$ and is a fixed point of $S$.

In general, there are three kinds of iterations of strong convergence that are used to approximate the fixed point of the nonlinear operator. The iterations are the Halpern iteration, the viscosity iteration, and the hybrid projection iteration. Recently, Hussain et al. [27] proposed a new surprising iteration that strongly converges to a fixed point of a nonexpansive mapping in Hilbert space. More precisely, the iteration is

$$
x_{1} \in H, \quad x_{n+1}=\alpha_{n}\left(1-\mu_{n}\right) x_{n}+\left(1-\alpha_{n}\right) T x_{n}, n \geq 1,
$$

where $H$ is a Hilbert space, $T: H \longrightarrow H$ is a nonexpansive mapping, and $\left\{\alpha_{n}\right\},\left\{\mu_{n}\right\} \subset(0,1]$ are the control sequences. The authors proved that $\left\{x_{n}\right\}$ generated by (5) strongly converges to a fixed point of $T$ under some certain conditions on $\left\{\alpha_{n}\right\}$ and $\left\{\mu_{n}\right\}$. Later on, Marino et al. [28] extended (5) to strict pseudocontraction.

In this paper, motivated by the work of $[22,26,27]$, we introduce some algorithms to solve a split common fixed point problem for two families of quasi- $\phi$-nonexpansive mappings in Banach spaces and prove the strong convergence for the proposed algorithms. As the application, we solve a split equilibrium problem in Banach space. Finally, we give a numerical example in infinite dimension Banach space to illustrate the main result of this paper. Our results extend the one of $\mathrm{Ma}$ et al. [22] from one quasi-nonexpansive mapping to two quasi-nonexpansive mappings and [27] from Hilbert space to Banach space.

\section{Preliminaries}

Let $E$ be a Banach space, and let $E^{*}$ be the dual space of $E$. For all $x \in E$ and $x^{*} \in E^{*}$, we denote the value of $x^{*}$ at $x$ by $\left\langle x, x^{*}\right\rangle$. The duality mapping $J$ on $E$ is defined by

$$
J(x)=\left\{x^{*} \in E^{*}:\left\langle x, x^{*}\right\rangle=\|x\|^{2}=\left\|x^{*}\right\|^{2}\right\}, \quad \forall x \in E .
$$

It is known that $J(x)$ is nonempty for all $x \in E$. A Banach space $E$ is said to be smooth if the limit 


$$
\lim _{n \longrightarrow \infty} \frac{\|x+t y\|-\|x\|}{t}
$$

exists for all $x, y \in S(E)=\{z \in E:\|z\|=1\}$. The space $E$ is smooth if and only if the duality mapping $J$ is single-valued.

A Banach space $E$ is said to be strictly convex if $(\| x+$ $y \| / 2)<1$ for $x, y \in E$ with $\|x\|=\|y\|=1$ and $x \neq y$ and uniformly convex if for each $\epsilon \in(0,2]$, there exists $\delta>0$ such that $(\|x+y\| / 2) \leq 1-\delta$ for all $x, y \in E$ with $\|x\|=\|y\|=1$ and $\|x-y\| \geq \epsilon$. It is known that if $E$ is smooth, strictly convex, and reflexive, then the duality mapping $J$ is singlevalued, one-to-one, and onto. Let $E$ be a smooth Banach space. The function $\phi: E \times E \longrightarrow \mathbb{R}$ is defined by

$$
\phi(x, y)=\|x\|^{2}-2\langle x, J y\rangle+\|y\|^{2},
$$

for all $x, y \in E$. From the definition of $\phi$, it is easy to see that, for all $x, y, z \in E$, the following hold: $\phi$

The following is an important property for the function

$$
\phi(x, y)=\phi(z, y)+\phi(x, z)+2\langle z-x, J y-J z\rangle,
$$

for all $x, y, z \in E$.

Lemma 1 (see [29]). Let $E$ be a uniformly convex and smooth Banach space, and let $\left\{x_{n}\right\}$ and $\left\{y_{n}\right\}$ be two sequences of E. If $\phi\left(x_{n}, y_{n}\right) \longrightarrow 0$ and either $\left\{x_{n}\right\}$ or $\left\{y_{n}\right\}$ is bounded, then $\left\|x_{n}-y_{n}\right\| \longrightarrow 0$.

For any bounded sequences $\left\{x_{n}\right\}$ and $\left\{y_{n}\right\}$ in a uniformly convex and uniformly smooth Banach space, the following hold:

$$
\phi\left(x_{n}, y_{n}\right) \longrightarrow 0 \Leftrightarrow\left\|x_{n}-y_{n}\right\| \longrightarrow 0 \Leftrightarrow\left\|J x_{n}-J y_{n}\right\| \longrightarrow 0
$$

Let $\Pi_{C}: E \longrightarrow C$ be mapping called the generalized projection [30] that assigns to an arbitrary element $x \in E$ the minimum point of the functional $\phi(x, y)$; that is, $\Pi_{C} x=\operatorname{argmin}_{y \in C} \phi(y, x)$.

Lemma 2 (see [30]). Let E be a smooth, strictly convex, and reflexive Banach space and $C$ be a nonempty closed convex subset of E. Then, the following conclusions hold:

(a) $\phi\left(x, \Pi_{C} y\right)+\phi\left(\Pi_{C} y, y\right) \leq \phi(x, y), \forall x \in C, \forall y \in E$

(b) For $x \in E, z=\Pi_{C} x$ if and only if $\langle z-y, J x-J z\rangle \geq 0$, $\forall y \in C$

(c) For $x, y \in E, \phi(x, y)=0$ if and only if $x=y$

Let $E$ be a strictly convex and reflexive Banach space and $C$ be a nonempty closed and convex subset. The metric projection

$$
P_{C} x=\operatorname{argmin}_{y \in C}\|y-x\|, \quad \forall x \in E .
$$

Lemma 3 (see [31]). Let $E$ be a smooth, strictly convex, and reflexive Banach space and $C$ be a nonempty, closed convex subset of E. Let $x \in E$. Then,

$$
z=P_{C} x \text { if and only if }\langle z-y, J(x-z)\rangle \geq 0, \quad \forall y \in C \text {. }
$$

Let $E$ be a strictly convex, smooth, and reflexive Banach space. The duality mapping $J^{*}$ from $E^{*}$ onto $E^{* *}=E$ coincides with the inverse of the duality mapping $J$ from $E$ onto $E^{*}$, that is, $J^{*}=J^{-1}$. Define a mapping $V: E \times E^{*} \longrightarrow \mathbb{R}[32]$ by

$$
V\left(x, x^{*}\right)=\|x\|^{2}-2\left\langle x, x^{*}\right\rangle+\left\|x^{*}\right\|^{2}, \quad \forall\left(x, x^{*}\right) \in E \times E^{*} .
$$

Lemma 4 (see [32]). Let $E$ be a reflexive, smooth, and strictly convex Banach space. Then,

$$
V\left(x, x^{*}\right) \leq V\left(x, x^{*}+y^{*}\right)-2\left\langle J^{-1} x^{*}-x, y^{*}\right\rangle,
$$

for all $x \in E$ and $x^{*}, y^{*} \in E^{*}$. Obviously, $V\left(x, x^{*}\right)=\phi\left(x, J^{-1} x^{*}\right)$ for all $x \in E$ and $x^{*} \in E$.

Let $E$ be a smooth Banach space. A mapping $T: E \longrightarrow E$ is said to be closed if for any sequence $\left\{x_{n}\right\} \subset E$ with $x_{n} \longrightarrow x$ and $T x_{n} \longrightarrow y$, then $T x_{n}=y . T$ is said to be quasi- $\phi$-nonexpansive mapping if $\operatorname{Fix}(T) \neq \varnothing$ and

$$
\phi(p, T x) \leq \phi(p, x)
$$

for all $p \in \operatorname{Fix}(T)$ and $x \in E$. For a quasi- $\phi$-nonexpansive mapping $T, \operatorname{Fix}(T)$ is convex. If $T$ is closed, then $\operatorname{Fix}(T)$ is closed, see [24].

Lemma 5 (see [33]). Let $r>0$. A real Banach space $E$ is uniformly convex if and only if there exists a continuous strictly increasing function $g:[0, \infty) \longrightarrow[0, \infty)$ with $g(0)=0$ such that

$$
\|t x+(1-t) y\|^{2} \leq t\|x\|^{2}+(1-t)\|y\|^{2}-t(1-t) g(\|x-y\|),
$$

for all $t \in[0,1]$ and $x, y \in B_{r}$, where $B_{r}=\{x \in E:\|x\| \leq r\}$.

Lemma 6 (see [33]). Let $r>0$. Let E be a 2-uniformly smooth Banach space with the best smoothness constants $k>0$. Then,

$$
\|x+y\|^{2} \leq\|x\|^{2}+2\langle y, J x\rangle+2 k^{2}\|y\|^{2},
$$


for all $x, y \in E$.

Lemma 7 (see [34]). Let $\left\{a_{n}\right\}$ be a sequence of nonnegative real numbers. Suppose that

$$
a_{n+1} \leq\left(1-\gamma_{n}\right) a_{n}+\gamma_{n} \delta_{n}, \quad \forall n \in \mathbb{N},
$$

where $\left\{\gamma_{n}\right\} \subset(0,1)$ and $\left\{\delta_{n}\right\} \subset \mathbb{R}$ satisfy the conditions:

$$
\begin{aligned}
\lim _{n \longrightarrow \infty} \gamma_{n} & =0, \\
\sum_{n=1}^{\infty} \gamma_{n} & =\infty, \text { and } \limsup _{\mathrm{n} \longrightarrow \infty} \delta_{\mathrm{n}} \leq 0 .
\end{aligned}
$$

Then, $\lim _{n \longrightarrow \infty} a_{n}=0$.

Lemma 8 (see [35]). Let $\left\{a_{n}\right\}$ be a sequence of real numbers such that there exists a subsequence $\left\{n_{i}\right\}$ of $\{n\}$ such that $a_{n_{i}}<a_{n_{i}+1}$ for all $i \in \mathbb{N}$. Then, there exists a nondecreasing sequence $\left\{m_{k}\right\} \subset \mathbb{N}$ such that $m_{k} \longrightarrow \infty$ as $k \longrightarrow \infty$, and the following properties are satisfied by all (sufficiently large) numbers $k \in \mathbb{N}$ :

$$
a_{m_{k}} \leq a_{m_{k}+1} \text { and } a_{k} \leq a_{m_{k}+1}
$$

In fact, $m_{k}=\max \left\{j \leq k: a_{j}<a_{j+1}\right\}$.

Lemma 9 (see [36]). Suppose that $\left\{a_{n}\right\}$ and $\left\{b_{n}\right\}$ are sequences of nonnegative real numbers such that

$$
a_{n+1} \leq a_{n}+b_{n}, \quad n \geq 1
$$

If $\sum_{n=1}^{\infty} b_{n}<\infty$, then $\lim _{n \longrightarrow \infty} a_{n}$ exists.

\section{Main Results}

In this section, let $E_{1}$ be a 2-uniformly convex and 2-uniformly smooth real Banach space with the best smoothness constant $k>0$ and $E_{2}$ be a uniformly smooth, strictly convex, and reflexive Banach space. Define the functions $\phi_{1}$ and $\phi_{2}$ by

$$
\begin{gathered}
\phi_{1}(x, y)=\|x\|_{1}^{2}-2\left\langle x, J_{1} y\right\rangle_{1}+\|y\|_{1}^{2}, \quad \forall x, y \in E_{1}, \\
\phi_{2}(u, v)=\|u\|_{2}^{2}-2\left\langle u, J_{2} v\right\rangle_{2}+\|v\|_{2}^{2}, \quad \forall u, v \in E_{2},
\end{gathered}
$$

where $\left\langle x, J_{1} y\right\rangle_{1}$ (resp., $\left\langle u, J_{1} v\right\rangle_{2}$ ) and $\|x\|_{1}$ (resp., $\|u\|_{2}$ ) denote the value of $J_{1} y$ at $x$ and norm of $x$ (resp., the value of $J_{1} v$ at $u$ and norm of $u$ ) in $E_{1}$ (resp. $E_{2}$ ), respectively. However, for convenience, we use the same symbols $\langle\cdot, \cdot\rangle$, $\|\cdot\|$, and $\phi$ in $E_{1}$ and $E_{2}$ without the confusion.

Let $A: E_{1} \longrightarrow E_{2}$ be a linear bounded operator with adjoint $A^{*}$. Let $S: E_{1} \longrightarrow E_{1}$ and $T: E_{2} \longrightarrow E_{2}$ be the quasi- $\phi$-nonexpansive mappings. Consider the following split common fixed point problem:

$$
\text { find } x \in \operatorname{Fix}(S) \text { such that } A x \in \operatorname{Fix}(T) \text {. }
$$

Denote the set of solutions of the above split common fixed point problem by $\Omega$. In this section, assume that $S$ and $T$ are closed and $I-S$ and $I-T$ are demiclosed at zeros in $E_{1}$ and $E_{2}$. Note that, from the closedness of $S$ and $T$, it follows that Fix $(S)$ and Fix $(T)$ are closed [24], which implies that $\Omega$ is closed. The convexity of $\Omega$ is from the convexity of Fix $(S)$. Assume that $\Omega$ is nonempty.

Let $x^{*}=\Pi_{\Omega} \theta$, where $\theta$ is the zero element in $E_{1}$. We will prove that sequence $\left\{x_{n}\right\}$ generated by the following algorithm converges strongly to $x^{*}$.

Algorithm 1. Take $x_{1} \in E_{1}$, and define a sequence $\left\{x_{n}\right\}$ by

$$
\left\{\begin{array}{l}
w_{n}=T A x_{n}, \\
Q_{n}=\left\{w \in E_{2}: \phi\left(w, w_{n}\right) \leq \phi\left(w, A x_{n}\right)\right\}, \\
z_{n}=J_{1}^{-1}\left(J_{1} x_{n}-\gamma_{n} A^{*} J_{2}\left(I-P_{Q_{n}}\right) A x_{n}\right), \\
y_{n}=J_{1}^{-1}\left(\beta_{n} J_{1} z_{n}+\left(1-\beta_{n}\right) J_{1} S z_{n}\right), \\
x_{n+1}=J_{1}^{-1}\left(\alpha_{n}\left(1-\tau_{n}\right) J_{1} x_{n}+\left(1-\alpha_{n}\right) J_{1} y_{n}\right), \quad n \geq 1,
\end{array}\right.
$$

where $\left\{\alpha_{n}\right\},\left\{\beta_{n}\right\} \subset(0,1),\left\{\tau_{n}\right\} \subset(\tau, 1)$ with $\tau \in(0,1)$ and

$$
\gamma_{n}= \begin{cases}\frac{\left\|\left(P_{Q_{n}}-I\right) A x_{n}\right\|^{2}}{2 k^{2}\left\|A^{*} J_{2}\left(I-P_{Q_{n}}\right) A x_{n}\right\|^{2}}, & \text { if }\left\|\left(P_{Q_{n}}-I\right) A x_{n}\right\| \neq 0, \\ 0, & \text { otherwise. }\end{cases}
$$

Lemma 10. The sequence $\left\{x_{n}\right\}$ is well-defined and bounded.

Proof. Since $\phi\left(w, w_{n}\right) \leq \phi\left(w, A x_{n}\right)$ is equivalent to $2\left\langle w, J_{2} A x_{n}-J_{2} w_{n}\right\rangle \leq\left\|A x_{n}\right\|^{2}-\left\|w_{n}\right\|^{2}$, it follows that $Q_{n}$ is closed and convex for each $n \geq 1$. For any $p \in \Omega$, it follows that $A p \in Q_{n}$ for all $n \geq 1$. Hence, each $Q_{n}$ is nonempty closed convex, which implies that $\left\{P_{Q_{n}} A x_{n}\right\}$ is well-defined. Now, we show that $\left\|\left(P_{Q_{n}}-I\right) A x_{n}\right\| \neq 0$ implies that $\left\|A^{*} J_{2}\left(P_{Q_{n}}-I\right) A x_{n}\right\| \neq 0$. Assume that $\left\|A^{*} J_{2}\left(P_{Q_{n}}-I\right) A x_{n}\right\|=$ 0 . We have $\left\langle A p-P_{Q_{n}} A x_{n}, J_{2}\left(P_{Q_{n}}-I\right) A x_{n}\right\rangle \geq 0$ by Lemma 3 and hence

$$
\begin{aligned}
0 & =\left\langle p-x_{n}, A^{*} J_{2}\left(P_{Q_{n}}-I\right) A x_{n}\right\rangle=\left\langle A p-A x_{n}, J_{2}\left(P_{Q_{n}}-I\right) A x_{n}\right\rangle \\
& =\left\langle A p-P_{Q_{n}} A x_{n}, J_{2}\left(P_{Q_{n}}-I\right) A x_{n}\right\rangle+\left\langle P_{Q_{n}} A x_{n}-A x_{n}, J_{2}\left(P_{Q_{n}}-I\right) A x_{n}\right\rangle \\
& =\left\langle A p-P_{Q_{n}} A x_{n}, J_{2}\left(P_{Q_{n}}-I\right) A x_{n}\right\rangle+\left\|\left(P_{Q_{n}}-I\right) A x_{n}\right\|^{2} \geq\left\|\left(P_{Q_{n}}-I\right) A x_{n}\right\|^{2} .
\end{aligned}
$$


It is a contradiction. It follows that $\left\|\left(P_{Q_{n}}-I\right) A x_{n}\right\| \neq 0$ implies that $\left\|A^{*} J_{2}\left(P_{Q_{n}}-I\right) A x_{n}\right\| \neq 0$. Hence, $\left\{z_{n}\right\}$ is welldefined. Furthermore, $\left\{x_{n}\right\}$ is well-defined.
Since $E_{1}$ is a 2-uniformly convex and 2-uniformly smooth real Banach space, $E_{1}^{*}$ is 2-uniformly smooth real Banach space, and $J_{1}=\left(J_{1}^{*}\right)^{-1}$. From (25) and Lemma 6, we have

$$
\begin{aligned}
\phi\left(x^{*}, z_{n}\right)= & \left\|x^{*}\right\|^{2}-2\left\langle x^{*}, J_{1} x_{n}+\gamma_{n} A^{*} J_{2}\left(P_{Q_{n}}-I\right) A x_{n}\right\rangle+\left\|J_{1} x_{n}+\gamma_{n} A^{*} J_{2}\left(P_{Q_{n}}-I\right) A x_{n}\right\|^{2} \\
\leq & \left\|x^{*}\right\|^{2}-2\left\langle x^{*}, J_{1} x_{n}\right\rangle-2 \gamma_{n}\left\langle x^{*}, A^{*} J_{2}\left(P_{Q_{n}}-I\right) A x_{n}\right\rangle+\left\|x_{n}\right\|^{2} \\
& +2 \gamma_{n}\left\langle x_{n}, A^{*} J_{2}\left(P_{Q_{n}}-I\right) A x_{n}\right\rangle+2 \gamma_{n}^{2} k^{2}\left\|A^{*} J_{2}\left(P_{Q_{n}}-I\right) A x_{n}\right\|^{2} \\
= & \phi\left(x^{*}, x_{n}\right)-2 \gamma_{n}\left\langle x^{*}-x_{n}, A^{*} J_{2}\left(P_{Q_{n}}-I\right) A x_{n}\right\rangle+2 \gamma_{n}^{2} k^{2}\left\|A^{*} J_{2}\left(P_{Q_{n}}-I\right) A x_{n}\right\|^{2} .
\end{aligned}
$$

Since $A x^{*} \in Q_{n},\left\langle A x^{*}-P_{Q_{n}} A x_{n}, J_{2}\left(P_{Q_{n}}-I\right) A x_{n}\right\rangle \geq 0$.

Hence, we have

$$
\begin{aligned}
2\left\langle x^{*}-x_{n}, A^{*} J_{2}\left(P_{Q_{n}}-I\right) A x_{n}\right\rangle & =2\left\langle A x^{*}-A x_{n}, J_{2}\left(P_{Q_{n}}-I\right) A x_{n}\right\rangle \\
& =2\left\langle A x^{*}-P_{Q_{n}} A x_{n}, J_{2}\left(P_{Q_{n}}-I\right) A x_{n}\right\rangle+2\left\|\left(P_{Q_{n}}-I\right) A x_{n}\right\|^{2} \geq 2\left\|\left(P_{Q_{n}}-I\right) A x_{n}\right\|^{2} .
\end{aligned}
$$

Combining (28) with (29), we obtain

$$
\begin{aligned}
\phi\left(x^{*}, z_{n}\right) & \leq \phi\left(x^{*}, x_{n}\right)-2 \gamma_{n}\left\|\left(P_{Q_{n}}-I\right) A x_{n}\right\|^{2}+2 \kappa^{2} \gamma_{n}^{2}\left\|A^{*} J_{2}\left(P_{Q_{n}}-I\right) A x_{n}\right\|^{2} \\
& =\phi\left(x^{*}, x_{n}\right)-\frac{\left\|\left(P_{Q_{n}}-I\right) A x_{n}\right\|^{4}}{2 k^{2}\left\|A^{*} J_{2}\left(P_{Q_{n}}-I\right) A x_{n}\right\|^{2}} \leq \phi\left(x^{*}, x_{n}\right) .
\end{aligned}
$$

Furthermore, by Lemma 5, (25), and (30) we obtain

$$
\begin{aligned}
\phi\left(x^{*}, y_{n}\right) & =\left\|x^{*}\right\|^{2}-2\left\langle x^{*}, \beta_{n} J_{1} z_{n}+\left(1-\beta_{n}\right) J_{1} S z_{n}\right\rangle+\left\|\beta_{n} J_{1} z_{n}+\left(1-\beta_{n}\right) J_{1} S z_{n}\right\|^{2} \\
& \leq\left\|x^{*}\right\|^{2}-2\left\langle x^{*}, \beta_{n} J_{1} z_{n}+\left(1-\beta_{n}\right) J_{1} S z_{n}\right\rangle+\beta_{n}\left\|z_{n}\right\|^{2}+\left(1-\beta_{n}\right)\left\|S z_{n}\right\|^{2}-\beta_{n}\left(1-\beta_{n}\right) g\left(\left\|J_{1} z_{n}-J_{1} S z_{n}\right\|\right) \\
& =\beta_{n} \phi\left(x^{*}, z_{n}\right)+\left(1-\beta_{n}\right) \phi\left(x^{*}, S z_{n}\right)-\beta_{n}\left(1-\beta_{n}\right) g\left(\left\|J_{1} z_{n}-J_{1} S z_{n}\right\|\right) \\
& \leq \phi\left(x^{*}, x_{n}\right)-\frac{\left\|\left(P_{Q_{n}}-I\right) A x_{n}\right\|^{4}}{2 k^{2}\left\|A^{*} J_{2}\left(P_{Q_{n}}-I\right) A x_{n}\right\|^{2}}-\beta_{n}\left(1-\beta_{n}\right) g\left(\left\|J_{1} z_{n}-J_{1} S z_{n}\right\|\right) .
\end{aligned}
$$

It follows from (25), (31), and Lemma 5 that 


$$
\begin{aligned}
\phi\left(x^{*}, x_{n+1}\right)= & \phi\left(x^{*}, J_{1}^{-1}\left(\alpha_{n}\left(1-\tau_{n}\right) J_{1} x_{n}+\left(1-\alpha_{n}\right) J_{1} y_{n}\right)\right)=\left\|x^{*}\right\|^{2}-2 \alpha_{n}\left(1-\tau_{n}\right)\left\langle x^{*}, J_{1} x_{n}\right\rangle-2\left(1-\alpha_{n}\right)\left\langle x^{*}, J_{1} y_{n}\right\rangle \\
& +\left\|\alpha_{n}\left(1-\tau_{n}\right) J_{1} x_{n}+\left(1-\alpha_{n}\right) J_{1} y_{n}\right\|^{2} \leq\left\|x^{*}\right\|^{2}-2 \alpha_{n}\left(1-\tau_{n}\right)\left\langle x^{*}, J_{1} x_{n}\right\rangle-2\left(1-\alpha_{n}\right)\left\langle x^{*}, J_{1} y_{n}\right\rangle \\
& +\alpha_{n}\left\|\left(1-\tau_{n}\right) J_{1} x_{n}\right\|^{2}+\left(1-\alpha_{n}\right)\left\|J_{1} y_{n}\right\|^{2} \leq\left\|x^{*}\right\|^{2}-2 \alpha_{n}\left(1-\tau_{n}\right)\left\langle x^{*}, J_{1} x_{n}\right\rangle-2\left(1-\alpha_{n}\right)\left\langle x^{*}, J_{1} y_{n}\right\rangle \\
& +\alpha_{n}\left(1-\tau_{n}\right)\left\|x_{n}\right\|^{2}+\left(1-\alpha_{n}\right)\left\|y_{n}\right\|^{2}=\alpha_{n}\left(1-\tau_{n}\right) \phi\left(x^{*}, x_{n}\right)+\left(1-\alpha_{n}\right) \phi\left(x^{*}, y_{n}\right)+\alpha_{n} \tau_{n}\left\|x^{*}\right\|^{2} \\
\leq & \alpha_{n}\left(1-\tau_{n}\right) \phi\left(x^{*}, x_{n}\right)+\left(1-\alpha_{n}\right)\left(\phi\left(x^{*}, x_{n}\right)-\frac{\left\|\left(P_{Q_{n}}-I\right) A x_{n}\right\|^{4}}{2 k^{2}\left\|A^{*} J_{2}\left(P_{Q_{n}}-I\right) A x_{n}\right\|^{2}}-\beta_{n}\left(1-\beta_{n}\right) g\left(\left\|J_{1} z_{n}-J_{1} S z_{n}\right\|\right)\right) \\
& +\alpha_{n} \tau_{n}\left\|x^{*}\right\|^{2} \\
= & \left(1-\alpha_{n} \tau_{n}\right) \phi\left(x^{*}, x_{n}\right)+\alpha_{n} \tau_{n}\left\|x^{*}\right\|^{2}-\left(1-\alpha_{n}\right)\left(\frac{\left\|\left(P_{Q_{n}}-I\right) A x_{n}\right\|^{4}}{2 k^{2}\left\|A^{*} J_{2}\left(P_{Q_{n}}-I\right) A x_{n}\right\|^{2}}+\beta_{n}\left(1-\beta_{n}\right) g\left(\left\|J_{1} z_{n}-J_{1} S z_{n}\right\|\right)\right) \\
\leq & \max \left\{\phi\left(x^{*}, x_{n}\right),\left\|x^{*}\right\|^{2}\right\} \leq \cdots \leq \max \left\{\phi\left(x^{*}, x_{1}\right),\left\|x^{*}\right\|^{2}\right\}, \quad n \geq 1 .
\end{aligned}
$$

So, $\left\{\phi\left(x^{*}, x_{n}\right)\right\}$ is bounded.

Lemma 11. Let $\left\{x_{n}\right\}$ be the sequence generated by Algorithm 1. Then,

$$
\begin{aligned}
\phi\left(x^{*}, x_{n+1}\right) \leq & \left(1-\alpha_{n} \tau_{n}\right) \phi\left(x^{*}, x_{n}\right)+2 \alpha_{n} \tau_{n}\left\langle x^{*}-x_{n+1}, J_{1} x^{*}\right. \\
& \left.+\left(1-\alpha_{n}\right)\left(J_{1} x_{n}-J_{1} y_{n}\right)\right\rangle .
\end{aligned}
$$

Proof. Let $h_{n}=\alpha_{n} J_{1} x_{n}+\left(1-\alpha_{n}\right) J_{1} y_{n}$. Then, by (31), we have

$$
\begin{aligned}
\phi\left(x^{*}, x_{n+1}\right)= & \phi\left(x^{*}, J_{1}^{-1}\left(\left(1-\alpha_{n} \tau_{n}\right) h_{n}+\alpha_{n} \tau_{n}\left(1-\alpha_{n}\right)\left(J_{1} y_{n}-J_{1} x_{n}\right)\right)\right) \\
= & V\left(x^{*},\left(1-\alpha_{n} \tau_{n}\right) h_{n}+\alpha_{n} \tau_{n}\left(1-\alpha_{n}\right)\left(J_{1} y_{n}-J_{1} x_{n}\right)\right) \\
\leq & V\left(x^{*},\left(1-\alpha_{n} \tau_{n}\right) h_{n}+\alpha_{n} \tau_{n}\left(1-\alpha_{n}\right)\left(J_{1} y_{n}-J_{1} x_{n}\right)+\alpha_{n} \tau_{n}\left(J_{1} x^{*}-\left(1-\alpha_{n}\right)\left(J_{1} y_{n}-J_{1} x_{n}\right)\right)\right) \\
& -2\left\langle x_{n+1}-x^{*}, \alpha_{n} \tau_{n}\left(J_{1} x^{*}-\left(1-\alpha_{n}\right)\left(J_{1} y_{n}-J_{1} x_{n}\right)\right)\right\rangle=V\left(x^{*},\left(1-\alpha_{n} \tau_{n}\right) h_{n}+\alpha_{n} \tau_{n} J_{1} x^{*}\right) \\
& -2\left\langle x_{n+1}-x^{*}, \alpha_{n} \tau_{n}\left(J_{1} x^{*}-\left(1-\alpha_{n}\right)\left(J_{1} y_{n}-J_{1} x_{n}\right)\right)\right\rangle \leq\left(1-\alpha_{n} \tau_{n}\right) \phi\left(x^{*}, J_{1}^{-1} h_{n}\right)+\alpha_{n} \tau_{n} \phi\left(x^{*}, x^{*}\right) \\
& -2\left\langle x_{n+1}-x^{*}, \alpha_{n} \tau_{n}\left(J_{1} x^{*}-\left(1-\alpha_{n}\right)\left(J_{1} y_{n}-J_{1} x_{n}\right)\right)\right\rangle \\
\leq & \left(1-\alpha_{n} \tau_{n}\right) \phi\left(x^{*}, x_{n}\right)+2 \alpha_{n} \tau_{n}\left\langle x^{*}-x_{n+1}, J_{1} x^{*}-\left(1-\alpha_{n}\right)\left(J_{1} y_{n}-J_{1} x_{n}\right)\right\rangle .
\end{aligned}
$$

Theorem 1 If the following conditions hold:

$$
\begin{aligned}
\lim _{n \longrightarrow \infty} \alpha_{n} & =0, \\
\sum_{n=1}^{\infty} \alpha_{n} & =\infty \text { and } \liminf _{n \rightarrow \infty} \beta_{n}\left(1-\beta_{n}\right)>0,
\end{aligned}
$$

then the sequence $\left\{x_{n}\right\}$ generated by Algorithm 1 converges strongly to the element $x^{*}$.

Proof. By (32), we have 


$$
\begin{aligned}
& \left(1-\alpha_{n}\right)\left(\frac{\left\|\left(P_{Q_{n}}-I\right) A x_{n}\right\|^{4}}{2 k^{2}\left\|A^{*} J_{2}\left(P_{Q_{n}}-I\right) A x_{n}\right\|^{2}}+\beta_{n}\left(1-\beta_{n}\right) g\left(\left\|J_{1} z_{n}-J_{1} S z_{n}\right\|\right)\right) \\
& \leq\left(1-\alpha_{n} \tau_{n}\right) \phi\left(x^{*}, x_{n}\right)-\phi\left(x^{*}, x_{n+1}\right)+\alpha_{n} \tau_{n}\left\|x^{*}\right\|^{2} \leq \phi\left(x^{*}, x_{n}\right)-\phi\left(x^{*}, x_{n+1}\right)+\alpha_{n} \tau_{n}\left\|x^{*}\right\|^{2} . \\
& \qquad \quad \lim _{n \rightarrow \infty}\left\|J_{1} z_{n}-J_{1} S z_{n}\right\|=0 .
\end{aligned}
$$
cases:

Now, we show that $\left\|x_{n}-x^{*}\right\| \longrightarrow 0$ by the following two

Case 1. Suppose that there exists $n_{0} \in \mathbb{N}$ such that $\left\{\phi\left(x^{*}, x_{n}\right)\right\}_{n=n_{0}}^{\infty}$ is nonincreasing. In this situation, $\left\{\phi\left(x^{*}, x_{n}\right)\right\}$ is convergent. By (37) and (38), we have $\lim _{n \longrightarrow \infty} \frac{\left\|\left(P_{Q_{n}}-I\right) A x_{n}\right\|^{4}}{\left\|A^{*} J_{2}\left(P_{Q_{n}}-I\right) A x_{n}\right\|^{2}}=\lim _{n \longrightarrow \infty} g\left(\left\|J_{1} z_{n}-J_{1} S z_{n}\right\|\right)=0$,
Since $\left\{\left\|A^{*} J_{2}\left(P_{Q_{n}}-I\right) A x_{n}\right\|\right\}$ is bounded, we have

$$
\lim _{n \longrightarrow \infty}\left\|\left(P_{Q_{n}}-I\right) A x_{n}\right\|=0 .
$$

By (40), we have

$$
\left\|J_{1} y_{n}-J_{1} z_{n}\right\|=\left(1-\beta_{n}\right)\left\|J_{1} z_{n}-J_{1} S z_{n}\right\| \longrightarrow 0 .
$$

Combining (39) with (42), we obtain

which implies that

$$
\begin{aligned}
\left\|J_{1} y_{n}-J_{1} x_{n}\right\| & \leq\left\|J_{1} y_{n}-J_{1} z_{n}\right\|+\left\|J_{1} z_{n}-J_{1} x_{n}\right\|=\left\|J_{1} y_{n}-J_{1} z_{n}\right\|+\gamma_{n}\left\|A^{*} J_{2}\left(P_{Q_{n}}-I\right) A x_{n}\right\| \\
& =\left\|J_{1} y_{n}-J_{1} z_{n}\right\|+\frac{\left\|\left(P_{Q_{n}}-I\right) A x_{n}\right\|^{2}}{2 \kappa^{2}\left\|A^{*} J_{2}\left(P_{Q_{n}}-I\right) A x_{n}\right\|} \longrightarrow 0 .
\end{aligned}
$$

On the contrary, from (25) and (43), it follows that

$$
\left\|J_{1} z_{n}-J_{1} x_{n+1}\right\| \leq\left\|J_{1} z_{n}-J_{1} y_{n}\right\|+\left\|J_{1} y_{n}-J_{1} x_{n+1}\right\|=\left\|J_{1} z_{n}-J_{1} y_{n}\right\|+\alpha_{n}\left\|\left(1-\tau_{n}\right) J_{1} x_{n}-J_{1} y_{n}\right\| \longrightarrow 0
$$

Since $E_{1}$ is a 2-uniformly convex and 2-uniformly smooth real Banach space, $J_{1}$ is uniformly norm-to-norm continuous. From (40), (42), and (44), it follows that

$\lim _{n \longrightarrow \infty}\left\|z_{n}-S z_{n}\right\|=\lim _{n \longrightarrow \infty}\left\|y_{n}-z_{n}\right\|=\lim _{n \longrightarrow \infty}\left\|z_{n}-x_{n+1}\right\|=0$.

Since $\left\{z_{n}\right\}$ is bounded, there exist a subsequence $\left\{z_{n_{k}}\right\}$ of $\left\{z_{n}\right\}$ converging weakly to $p \in E_{1}$ such that

$$
\begin{aligned}
\limsup _{n \longrightarrow \infty}\left\langle x^{*}-z_{n}, J_{1} x^{*}\right\rangle & =\lim _{k \longrightarrow \infty}\left\langle x^{*}-z_{n_{k^{*}}}, J_{1} x^{*}\right\rangle \\
& =\left\langle x^{*}-p, J_{1} x^{*}\right\rangle .
\end{aligned}
$$

Now, we show that $p \in \Omega$. First, by (45) and demicloseness principle at zero of $S$, we have $p \in \operatorname{Fix}(S)$. On the contrary, since $P_{\mathrm{Q}_{n}} A x_{n} \in Q_{n} \quad$ and $\left\|P_{Q_{n}} A x_{n}-A x_{n}\right\| \longrightarrow 0$, we have

$$
\phi\left(P_{\mathrm{Q}_{n}} A x_{n}, w_{n}\right) \leq \phi\left(P_{\mathrm{Q}_{n}} A x_{n}, A x_{n}\right) \longrightarrow 0 .
$$

By Lemma 1, it follows that

$$
\left\|P_{Q_{n}} A x_{n}-w_{n}\right\|=\left\|P_{Q_{n}} A x_{n}-T A x_{n}\right\| \longrightarrow 0 .
$$

Hence,

$\left\|A x_{n}-T A x_{n}\right\| \leq\left\|A x_{n}-P_{Q_{n}} A x_{n}\right\|+\left\|P_{Q_{n}} A x_{n}-T A x_{n}\right\| \longrightarrow 0$.

Since $A$ is bounded and linear, by (45), we can conclude that $\left\{A x_{n_{k}+1}\right\}$ converges weakly to $A p \in E_{2}$. By (49) and demi-closedness principle of $T$, we obtain that $A p \in \operatorname{Fix}(T)$. Hence, $p \in \Omega$. Therefore, by (45) and Lemma 3,

$$
\begin{aligned}
\limsup _{n \longrightarrow \infty}\left\langle x^{*}-x_{n+1}, J_{1} x^{*}\right\rangle & =\limsup _{n \longrightarrow \infty}\left\langle x^{*}-z_{n}, J_{1} x^{*}\right\rangle \\
& =\left\langle x^{*}-p, J_{1} x^{*}\right\rangle \leq 0 .
\end{aligned}
$$

Finally, the conclusion $\left\|x_{n}-x^{*}\right\| \longrightarrow 0$ follows from the hypothesis on $\left\{\alpha_{n}\right\},(33),(43),(50)$, and Lemma 4. Case 2. Suppose that there exists a subsequence $\left\{n_{i}\right\}$ of $\{n\}$ such that 


$$
\phi\left(x^{*}, x_{n_{i}}\right)<\phi\left(x^{*}, x_{n_{i}+1}\right)
$$

for all $i \in \mathbb{N}$.

Then, by Lemma 5 , there exists a nondecreasing sequence $\left\{m_{k}\right\} \subset \mathbb{N}$ such that $m_{k} \longrightarrow \infty$ :

$$
\begin{aligned}
\phi\left(x^{*}, x_{m_{k}}\right) & \leq \phi\left(x^{*}, x_{m_{k}+1}\right) \text { and } \phi\left(x^{*}, x_{k}\right) \\
& \leq \phi\left(x^{*}, x_{m_{k}+1}\right), \quad \forall k \geq 1 .
\end{aligned}
$$

Replacing $n$ with $m_{k}$ in (38), by (52), we have

$$
\begin{aligned}
& \left(1-\alpha_{m_{k}}\right)\left(\frac{\left\|\left(P_{Q_{m_{k}+1}}-I\right) A x_{m_{k}}\right\|^{4}}{2 k^{2}\left\|A^{*} J_{2}\left(P_{Q_{m_{k}+1}}-I\right) A x_{m_{k}}\right\|^{2}}+\beta_{m_{k}}\left(1-\beta_{m_{k}}\right) g\left(\left\|J_{1} z_{m_{k}}-J_{1} S z_{m_{k}}\right\|\right)\right) \\
& \leq \phi\left(x^{*}, x_{m_{k}}\right)-\phi\left(x^{*}, x_{m_{k}+1}\right)+\alpha_{m_{k}} \tau_{m_{k}}\left\|x^{*}\right\|^{2} \leq \alpha_{m_{k}} \tau_{m_{k}}\left\|x^{*}\right\|^{2} .
\end{aligned}
$$

Then, by a similar process with proving (43)-(50), we can obtain that

$\lim _{k \longrightarrow \infty}\left\|J_{1} x_{m_{k}}-J_{1} y_{m_{k}}\right\|=0$ and $\limsup _{n \longrightarrow \infty}\left\langle x^{*}-x_{m_{k}+1}, J_{1} x^{*}\right\rangle \leq 0$.

Replacing $n$ with $m_{k}$ in (33), we have

$$
\begin{aligned}
\phi\left(x^{*}, x_{m_{k}+1}\right) \leq & \left(1-\alpha_{m_{k}} \tau_{m_{k}}\right) \phi\left(x^{*}, x_{m_{k}}\right) \\
& +2 \alpha_{m_{k}} \tau_{m_{k}}\left\langle x^{*}-x_{m_{k}+1}, J_{1} x^{*}\right. \\
& \left.+\left(1-\alpha_{m_{k}}\right)\left(J_{1} y_{m_{k}}-J_{1} x_{m_{k}}\right)\right\rangle,
\end{aligned}
$$

from which we obtain

$$
\begin{aligned}
\alpha_{m_{k}} \tau_{m_{k}} \phi\left(x^{*}, x_{m_{k}}\right) & \leq \phi\left(x^{*}, x_{m_{k}}\right)-\phi\left(x^{*}, x_{m_{k}+1}\right)+2 \alpha_{m_{k}} \tau_{m_{k}}\left\langle x^{*}-x_{m_{k}+1}, J_{1} x^{*}+\left(1-\alpha_{m_{k}}\right)\left(J_{1} y_{m_{k}}-J_{1} x_{m_{k}}\right)\right\rangle \\
& \leq 2 \alpha_{m_{k}} \tau_{m_{k}}\left\langle x^{*}-x_{m_{k}+1}, J_{1} x^{*}+\left(1-\alpha_{m_{k}}\right)\left(J_{1} y_{m_{k}}-J_{1} x_{m_{k}}\right)\right\rangle .
\end{aligned}
$$

Since $\alpha_{m_{k}} \tau_{m_{k}}>0$, by (54) and (56), we have

$$
\begin{aligned}
\phi\left(x^{*}, x_{m_{k}}\right) \leq & 2\left\langle x^{*}-x_{m_{k}+1}, J_{1} x^{*}\right. \\
+ & \left.\left(1-\alpha_{m_{k}}\right)\left(J_{1} y_{m_{k}}-J_{1} x_{m_{k}}\right)\right\rangle \longrightarrow 0 .
\end{aligned}
$$

Furthermore, by (54), (55), and (57), it follows that

$$
\lim _{k \rightarrow \infty} \phi\left(x^{*}, x_{m_{k}+1}\right)=0 \text {. }
$$

However, $\phi\left(x^{*}, x_{k}\right) \leq\left\|x_{m_{k}+1}-x^{*}\right\|$ for all $k \geq 1$. So, we conclude that $\phi\left(x^{*}, x_{k}\right) \longrightarrow 0$ as $k \longrightarrow \infty$ and hence $\left\|x_{k}-x^{*}\right\| \longrightarrow 0$ as $k \longrightarrow \infty$ by Lemma 1 . The proof is complete.

Remark 1. If $\left\|\left(P_{Q_{n}}-I\right) A x_{n}\right\|=0$ for all $n \geq 1$, then $\gamma_{n}=0$ and $z_{n}=x_{n}$ for all $n \geq 1$. In this case, $A x_{n}=P_{Q_{n}} A x_{n}$ and $\phi\left(A x_{n}, w_{n}\right)=\phi\left(A x_{n}, T A x_{n}\right) \leq \phi\left(A x_{n}, A x_{n}\right)=0$, which implies that $A x_{n}=T A x_{n}$ for all $n \geq 1$. The iterative scheme (25) becomes

$$
\left\{\begin{array}{l}
y_{n}=J_{1}^{-1}\left(\beta_{n} J_{1} x_{n}+\left(1-\beta_{n}\right) J_{1} S x_{n}\right) \\
x_{n+1}=J_{1}^{-1}\left(\alpha_{n}\left(1-\tau_{n}\right) J_{1} x_{n}+\left(1-\alpha_{n}\right) J_{1} y_{n}\right), \quad n \geq 1
\end{array}\right.
$$

By the proof process above, we still can see that $\left\{x_{n}\right\}$ converges strongly to $x^{*}=P_{\mathrm{Fix}(S)} \theta$. Since $A$ is linear and bounded, $A x_{n} \longrightarrow A x^{*}$, which implies that $A x_{n} \rightarrow x^{*}$. Note that $A x_{n}=T A x_{n}$, for all $n \geq 1$, and $A x_{n}-T A x_{n} \longrightarrow 0$ as $n \longrightarrow \infty$. By the hypothesis that $I-T$ is demi-closedness at zero, we get $A x^{*}=T A x^{*}$. Hence, $x^{*} \in \Omega$. Hence, without loss generality, we assume that $\gamma_{n} \neq 0$ for all $n \geq 1$ in the proof process.

Algorithm 2. Take $u=x_{1} \in E_{1}$, and define a sequence $\left\{x_{n}\right\}$ by

$$
\left\{\begin{array}{l}
w_{n}=T A x_{n}, \\
Q_{n}=\left\{w \in E_{2}: \phi\left(w, w_{n}\right) \leq \phi\left(w, A x_{n}\right)\right\}, \\
z_{n}=J_{1}^{-1}\left(J_{1} x_{n}-\gamma_{n} A^{*} J_{2}\left(I-P_{Q_{n}}\right) A x_{n}\right), \\
y_{n}=J_{1}^{-1}\left(\beta_{n} J_{1} z_{n}+\left(1-\beta_{n}\right) J_{1} S z_{n}\right), \\
x_{n+1}=J_{1}^{-1}\left(\alpha_{n} J_{1} u+\left(1-\alpha_{n}\right) J_{1} y_{n}\right), \quad n \geq 1,
\end{array}\right.
$$

where $\left\{\alpha_{n}\right\},\left\{\beta_{n}\right\} \subset(0,1)$ and

$$
\gamma_{n}= \begin{cases}\frac{\left\|\left(P_{Q_{n}}-I\right) A x_{n}\right\|^{2}}{2 k^{2}\left\|A^{*} J_{2}\left(I-P_{Q_{n}}\right) A x_{n}\right\|^{2}}, & \left\|\left(P_{Q_{n}}-I\right) A x_{n}\right\| \neq 0, \\ 0, & \text { otherwise. }\end{cases}
$$


Lemma 12. $\left\{x_{n}\right\}$ is well-defined and bounded.

Proof. By a similar proof lines of Lemma 10, we can show that $\left\{x_{n}\right\}$ is well-defined. Now, we prove that $\left\{x_{n}\right\}$ is bounded. By (29)-(31), (60), and Lemma 5, for any $\widehat{x} \in \Omega$, we have

$$
\begin{aligned}
\phi\left(\hat{x}, x_{n+1}\right)= & \phi\left(\hat{x}, J_{1}^{-1}\left(\alpha_{n} J_{1} u+\left(1-\alpha_{n}\right) J_{1} y_{n}\right)\right)=\|\hat{x}\|^{2}-2 \alpha_{n}\left\langle\hat{x}, J_{1} u\right\rangle-2\left(1-\alpha_{n}\right)\left\langle\hat{x}, J_{1} y_{n}\right\rangle \\
& +\left\|\alpha_{n} J_{1} u+\left(1-\alpha_{n}\right) J_{1} y_{n}\right\|^{2} \leq\|\hat{x}\|^{2}-2 \alpha_{n}\left\langle\hat{x}, J_{1} u\right\rangle-2\left(1-\alpha_{n}\right)\left\langle\hat{x}, J_{1} y_{n}\right\rangle \\
& +\alpha_{n}\|u\|^{2}+\left(1-\alpha_{n}\right)\left\|y_{n}\right\|^{2}=\alpha_{n} \phi(\hat{x}, u)+\left(1-\alpha_{n}\right) \phi\left(\widehat{x}, y_{n}\right) \leq \alpha_{n} \phi(\widehat{x}, u)+\left(1-\alpha_{n}\right) \\
& \cdot\left(\phi\left(\widehat{x}, x_{n}\right)-\frac{\left\|\left(P_{Q_{n}}-I\right) A x_{n}\right\|^{4}}{2 k^{2}\left\|A^{*} J_{2}\left(P_{Q_{n}}-I\right) A x_{n}\right\|^{2}}-\beta_{n}\left(1-\beta_{n}\right) g\left(\left\|J_{1} z_{n}-J_{1} S z_{n}\right\|\right)\right) \\
\leq & \alpha_{n} \phi(\hat{x}, u)+\left(1-\alpha_{n}\right) \phi\left(\widehat{x}, x_{n}\right) \leq \alpha_{n} \phi(\widehat{x}, u)+\phi\left(\hat{x}, x_{n}\right), \quad n \geq 1 .
\end{aligned}
$$

By the hypothesis on $\left\{\alpha_{n}\right\}$ and Lemma 9, it follows that the limit of $\left\{\phi\left(\hat{x}, x_{n}\right)\right\}$ exists. Hence, $\left\{x_{n}\right\}$ is bounded.

Theorem 2. Assume that $S$ and $T$ are closed. If the interior of $\Omega$ is nonempty and $\left\{\alpha_{n}\right\}$ and $\left\{\beta_{n}\right\}$ satisfy the following conditions

$$
\sum_{n=1}^{\infty} \alpha_{n}<\infty \text { and } \liminf _{n \longrightarrow \infty} \beta_{n}\left(1-\beta_{n}\right)>0,
$$

then $\left\{x_{n}\right\}$ generated by Algorithm 2 converges strongly to the element $x^{*}=\lim _{n \longrightarrow \infty} \Pi_{\Omega} x_{n}$.

Proof. We first show that $\left\{x_{n}\right\}$ is a Cauchy sequence and hence converges strongly to some point $x^{*} \in E_{1}$. Since the interior of $\Omega$ is nonempty, there exist $p \in \Omega$ and $r>0$ such that

$$
p+r h \in \Omega
$$

whenever $\|h\| \leq 1$. By (10), we have

$$
\begin{aligned}
\phi\left(p, x_{n}\right) & =\phi\left(x_{n+1}, x_{n}\right)+\phi\left(p, x_{n+1}\right)+2\left\langle x_{n+1}-p, J_{1} x_{n}-J_{1} x_{n+1}\right\rangle \\
& =\phi\left(x_{n+1}, x_{n}\right)+\phi\left(p, x_{n+1}\right)+2\left\langle x_{n+1}-(p+r h), J_{1} x_{n}-J_{1} x_{n+1}\right\rangle+2 r\left\langle h, J_{1} x_{n}-J_{1} x_{n+1}\right\rangle
\end{aligned}
$$

On the contrary, by (10), again we have

$$
\begin{aligned}
\phi\left(p+r h, x_{n}\right)= & \phi\left(x_{n+1}, x_{n}\right)+\phi\left(p+r h, x_{n+1}\right) \\
& +2\left\langle x_{n+1}-(p+r h), J_{1} x_{n}-J_{1} x_{n+1}\right\rangle .
\end{aligned}
$$

$$
\begin{aligned}
2 r\left\langle h, J_{1} x_{n}-J_{1} x_{n+1}\right\rangle & =\phi\left(p, x_{n}\right)-\left(\phi\left(x_{n+1}, x_{n}\right)+\phi\left(p, x_{n+1}\right)+2\left\langle x_{n+1}-(p+r h), J_{1} x_{n}-J_{1} x_{n+1}\right\rangle\right) \\
& =\phi\left(p, x_{n}\right)-\phi\left(x_{n+1}, x_{n}\right)-\phi\left(p, x_{n+1}\right)-\phi\left(p+r h, x_{n}\right)+\phi\left(x_{n+1}, x_{n}\right)+\phi\left(p+r h, x_{n+1}\right) \\
& =\frac{1}{2 r}\left(\phi\left(p, x_{n}\right)-\phi\left(p, x_{n+1}\right)\right)+\phi\left(p+r h, x_{n+1}\right)-\phi\left(p+r h, x_{n}\right) .
\end{aligned}
$$

Since $p+r h \in \Omega$, from (62) and (67), it follows that 


$$
\begin{aligned}
2 r\left\langle h, J_{1} x_{n}-J_{1} x_{n+1}\right\rangle & \leq \phi\left(p, x_{n}\right)-\phi\left(p, x_{n+1}\right)+\alpha_{n}\left(\phi(p+r h, u)-\phi\left(p+r h, x_{n}\right)\right) \\
& \leq \phi\left(p, x_{n}\right)-\phi\left(p, x_{n+1}\right)+\alpha_{n} \phi(p+r h, u) .
\end{aligned}
$$

Since $h$ with $\|h\| \leq 1$ is arbitrary, we have

So, for all $m>n$,

$$
\begin{aligned}
\left\|J_{1} x_{n}-J_{1} x_{n+1}\right\| \leq & \frac{1}{2 r}\left(\phi\left(p, x_{n}\right)-\phi\left(p, x_{n+1}\right)\right. \\
& \left.+\alpha_{n} \phi(p+r h, u)\right) .
\end{aligned}
$$

$$
\begin{aligned}
\left\|J_{1} x_{n}-J_{1} x_{m}\right\| & =\left\|J_{1} x_{n}-J_{1} x_{n+1}+J_{1} x_{n+1}-\cdots-J_{1} x_{m-1}+J_{1} x_{m-1}+J_{1} x_{m}\right\| \\
& \leq \sum_{i=n}^{m-1}\left\|J_{1} x_{i}-J_{1} x_{i+1}\right\| \leq \frac{1}{2 r} \sum_{i=n}^{m-1}\left(\phi\left(p, x_{i}\right)-\phi\left(p, x_{i+1}\right)+\alpha_{i} \phi(p+r h, u)\right) \\
& =\frac{1}{2 r} \sum_{i=n}^{m-1}\left(\phi\left(p, x_{i}\right)-\phi\left(p, x_{i+1}\right)\right)+\frac{\phi(p+r h, u)}{2 r} \sum_{i=n}^{m-1} \alpha_{i}=\phi\left(p, x_{n}\right)-\phi\left(p, x_{m}\right)+\frac{\phi(p+r h, u)}{2 r} \sum_{i=n}^{m-1} \alpha_{i} .
\end{aligned}
$$

Since the limit of $\left\{\phi\left(p, x_{n}\right)\right\}$ exists and $\sum_{n=1}^{\infty} \alpha_{n}<\infty$, from (70), we see

$$
\lim _{m, n \longrightarrow \infty}\left\|J_{1} x_{n}-J_{1} x_{m}\right\|=0,
$$

which implies that $\left\{J_{1} x_{n}\right\}$ is a Cauchy sequence in $E_{1}^{*}$. Hence, $\left\{J_{1} x_{n}\right\}$ converges strongly to some point in $E_{1}^{*}$. Since $E_{1}^{*}$ has a Fréchet differentiable norm, then $J_{1}^{-1}$ is continuous on $E_{1}^{*}$. Hence, $x_{n}$ converges strongly to some point $x^{*}$ in $E_{1}$.

For any $\hat{x} \in \Omega$, by (62), we have

$$
\begin{aligned}
& \left(1-\alpha_{n}\right)\left(\frac{\left\|\left(P_{Q_{n}}-I\right) A x_{n}\right\|^{4}}{2 k^{2}\left\|A^{*} J_{2}\left(P_{Q_{n}}-I\right) A x_{n}\right\|^{2}}+\beta_{n}\left(1-\beta_{n}\right) g\left(\left\|J_{1} z_{n}-J_{1} S z_{n}\right\|\right)\right) \\
& \leq \alpha_{n} \phi(\widehat{x}, u)+\left(1-\alpha_{n}\right) \phi\left(\hat{x}, x_{n}\right)-\phi\left(\widehat{x}, x_{n+1}\right) \leq \alpha_{n} \phi(\hat{x}, u)+\phi\left(\widehat{x}, x_{n}\right)-\phi\left(\widehat{x}, x_{n+1}\right) .
\end{aligned}
$$

Since the limit of $\left\{\phi\left(\widehat{x}, x_{n}\right)\right\}$ exists, by the hypothesis on $\left\{\alpha_{n}\right\}$ and $\left\{\beta_{n}\right\}$, it follows that

$$
\lim _{n \longrightarrow \infty} \frac{\left\|\left(P_{Q_{n}}-I\right) A x_{n}\right\|^{4}}{\left\|A^{*} J_{2}\left(P_{Q_{n}}-I\right) A x_{n}\right\|^{2}}=\lim _{n \longrightarrow \infty} g\left(\left\|J_{1} z_{n}-J_{1} S z_{n}\right\|\right)=0,
$$

which implies that

$$
\lim _{n \longrightarrow \infty}\left\|\left(P_{Q_{n}}-I\right) A x_{n}\right\|=0 \text { and } \lim _{n \longrightarrow \infty}\left\|J_{1} z_{n}-J_{1} S z_{n}\right\|=0,
$$

and hence

$$
\left\|z_{n}-S z_{n}\right\| \longrightarrow 0 .
$$

On the contrary, by (60) and (73), we have

$$
\left\|J_{1} z_{n}-J_{1} x_{n}\right\|=\gamma_{n}\left\|A^{*} J_{2}\left(I-P_{Q_{n}}\right) A x_{n}\right\|
$$

$$
=\frac{\left\|\left(I-P_{Q_{n}}\right) A x_{n}\right\|^{2}}{\left\|A^{*} J_{2}\left(I-P_{Q_{n}}\right) A x_{n}\right\|} \longrightarrow 0 .
$$

It follows that

$$
\left\|z_{n}-x_{n}\right\| \longrightarrow 0
$$

Hence, $\left\{z_{n}\right\}$ converges strongly to $x^{*} \in E_{1}$. Since $S$ is closed, by (75), we get $x^{*}=S x^{*}$.

Now, we show that $A x^{*}=T A x^{*}$. From (49), it follows that $\left\|A x_{n}-T A x_{n}\right\| \longrightarrow 0$. Since $A$ is linear bounded, $A x_{n} \longrightarrow A x^{*}$. From the closedness of $T$, we get $A x^{*}=T A x^{*}$. Therefore, $x^{*} \in \Omega$. Finally, we show that $x^{*}=\lim _{n \longrightarrow \infty} \Pi_{\Omega} x_{n}$. In fact, since $x^{*} \in \Omega$, by Lemma 2 , we have

$$
\phi\left(x^{*}, \Pi_{\Omega} x_{n}\right) \leq \phi\left(x^{*}, x_{n}\right) \longrightarrow 0 .
$$


It follows that $x^{*}=\lim _{n \longrightarrow \infty} \Pi_{\Omega} x_{n}$. The proof is complete.

Let $Q$ be a nonempty closed convex subset of $E_{2}$. In Algorithms 1 and 2, if putting $T=I$ and $Q_{1}=Q$, we have $w_{n}=A x_{n}$ and $Q_{n}=Q$ for all $n \geq 1$. Then, we have the following results.

Corollary 1. Let $E_{1}$ be a 2-uniformly convex and 2-uniformly smooth real Banach space with the best smoothness constant $k>0$ and $E_{2}$ be a uniformly smooth, strictly convex, and reflexive Banach space with a nonempty closed convex subset $Q \subset E_{2}$. Let $A: E_{1} \longrightarrow E_{2}$ be a linear bounded operator with adjoint $A^{*}$. Let $S: E_{1} \longrightarrow E_{1}$ and $Q \subset E_{2}$ be a nonempty subset. Assume that $I-S$ is demi-closedness at zero and $\Gamma \neq \varnothing$, where $\Gamma=\left\{x \in E_{1}: x \in \operatorname{Fix}(S), A x \in Q\right\}$. Let $x_{1} \in E_{1}$ and define a sequence $\left\{x_{n}\right\}$ by

$$
\left\{\begin{array}{l}
z_{n}=J_{1}^{-1}\left(J_{1} x_{n}-\gamma_{n} A^{*} J_{2}\left(I-P_{\mathrm{Q}}\right) A x_{n}\right), \\
y_{n}=J_{1}^{-1}\left(\beta_{n} J_{1} z_{n}+\left(1-\beta_{n}\right) J_{1} S z_{n}\right), \\
x_{n+1}=J_{1}^{-1}\left(\alpha_{n}\left(1-\tau_{n}\right) J_{1} x_{n}+\left(1-\alpha_{n}\right) J_{1} y_{n}\right), \quad n \geq 1,
\end{array}\right.
$$

where $\left\{\alpha_{n}\right\},\left\{\beta_{n}\right\} \subset(0,1),\left\{\tau_{n}\right\} \subset(\tau, 1)$ with $\tau \in(0,1)$ and

$$
\gamma_{n}= \begin{cases}\frac{\left\|\left(P_{Q_{n}}-I\right) A x_{n}\right\|^{2}}{2 k^{2}\left\|A^{*} J_{2}\left(I-P_{Q_{n}}\right) A x_{n}\right\|^{2}}, & \left\|\left(P_{Q_{n}}-I\right) A x_{n}\right\| \neq 0, \\ 0, & \text { otherwise. }\end{cases}
$$

If the following conditions hold,

$$
\begin{aligned}
\lim _{n \longrightarrow \infty} \alpha_{n} & =0, \\
\sum_{n=1}^{\infty} \alpha_{n} & =\infty \text { and } \liminf _{n \longrightarrow \infty} \beta_{n}\left(1-\beta_{n}\right)>0,
\end{aligned}
$$

then the sequence $\left\{x_{n}\right\}$ generated by (60) converges strongly to the element $x^{*}=\Pi_{\Gamma} \theta$, where $\theta$ is the zero element in $E_{1}$.

Corollary 2. Let $E_{1}$ be a 2-uniformly convex and 2-uniformly smooth real Banach space with the best smoothness constant $k>0$ and $E_{2}$ be a uniformly smooth, strictly convex, and reflexive Banach space with a nonempty closed convex subset $Q \subset E_{2}$. Let $A: E_{1} \longrightarrow E_{2}$ be a linear bounded operator with adjoint $A^{*}$. Let $S: E_{1} \longrightarrow E_{1}$ and $Q \subset E_{2}$ be a nonempty subset. Assume that $S$ is closed and the interior of $\Gamma$ is nonempty, where $\Gamma=\left\{x \in E_{1}: x \in\right.$ Fix $\left.(S), A x \in Q\right\}$. Let $u=$ $x_{1} \in E_{1}$ and define a sequence $\left\{x_{n}\right\}$ by

$$
\left\{\begin{array}{l}
z_{n}=J_{1}^{-1}\left(J_{1} x_{n}-\gamma_{n} A^{*} J_{2}\left(I-P_{Q}\right) A x_{n}\right), \\
y_{n}=J_{1}^{-1}\left(\beta_{n} J_{1} z_{n}+\left(1-\beta_{n}\right) J_{1} S z_{n}\right), \\
x_{n+1}=J_{1}^{-1}\left(\alpha_{n} J_{1} u+\left(1-\alpha_{n}\right) J_{1} y_{n}\right), n \geq 1,
\end{array}\right.
$$

where $\left\{\alpha_{n}\right\},\left\{\beta_{n}\right\} \subset(0,1)$ and $\gamma_{n}=\left\{\left\|\left(P_{Q_{n}}-I\right) A x_{n}\right\|^{2} / 2 k^{2} \| A^{*}\right.$ $J_{2}\left(I-P_{Q_{n}}\right) A x_{n}\left\|^{2},\right\|\left(P_{Q_{n}}-I\right) A x_{n} \| \neq 0,0$, otherwise.

If the following conditions hold

$$
\begin{aligned}
& \lim _{n \longrightarrow \infty} \alpha_{n}=0, \\
& \sum_{n=1}^{\infty} \alpha_{n}<\infty \text { and } \liminf _{n \longrightarrow \infty} \beta_{n}\left(1-\beta_{n}\right)>0,
\end{aligned}
$$

then the sequence $\left\{x_{n}\right\}$ generated by (82) converges strongly to some element $x^{*}=\lim _{n \longrightarrow \infty} \Pi_{\Gamma} x_{n}$.

\section{Application}

Let $E_{1}$ and $E_{2}$ be two Banach spaces and $f_{1}: E_{1} \times E_{1} \longrightarrow \mathbb{R}$ and $f_{2}: E_{2} \times E_{2} \longrightarrow \mathbb{R}$ be the bifunctions. Let $A: E_{1} \longrightarrow E_{2}$ be a linear bounded operator. In this section, we consider a split equilibrium problem: find a point $x^{*} \in E_{1}$ such that

$$
x^{*} \in \operatorname{EP}\left(f_{1}\right) \text { and } A x^{*} \in \operatorname{EP}\left(f_{2}\right),
$$

where $\operatorname{EP}\left(f_{1}\right)=\left\{x \in E_{1}: f_{1}(x, y) \geq 0, \forall y \in E_{1}\right\} \quad$ and $\operatorname{EP}\left(f_{2}\right)=\left\{u \in E_{2}: f_{2}(u, v) \geq 0, \forall v \in E_{2}\right\}$. We denote the set of solution of problem (84) by $\Lambda$. That is, $\Lambda=\left\{x \in \operatorname{EP}\left(f_{1}\right): A x \in \operatorname{EP}\left(f_{2}\right)\right\}$.

The split equilibrium problem has been studied by many authors in Hilbert space, see [37-41]. However, few results on the split equilibrium problem in Banach space is reported by far.

Lemma 13 (see [24]). Let $E$ be a strictly convex, reflexive, and uniform smooth Banach space and $f: E \times E \longrightarrow \mathbb{R}$ be a bifunction satisfying the following conditions:

(A1) $f(x, x)=0$ for all $x \in E$.

(A2) $f$ is monotone, i.e., $f(x, y)+f(y, x) \leq 0$ for all $x, y \in E$.

(A3) For all $x, y, z \in E$,

$$
\limsup _{t \longrightarrow 0^{+}} f(t z+(1-t) x, y) \leq f(x, y) .
$$

(A4) For all $x \in E, f(x, \cdot)$ is convex and lower semicontinuous.

For $r>0$ and $x \in E$, define a mapping $T_{r}: E \longrightarrow E$ as follows:

$$
\begin{aligned}
\mathrm{T}_{r}^{f} x & =\left\{z \in E: f(z, y)+\frac{1}{r}\langle y-z, J z-J x\rangle\right. \\
& \geq 0 \text { for all } y \in E\},
\end{aligned}
$$

for all $x \in E$. Then, the following hold:

(1) $\mathrm{T}_{r}^{f}$ is single-valued

(2) $\operatorname{Fix}\left(\mathrm{T}_{r}^{f}\right)=E P(f)$

(3) $E P(f)$ is closed and convex 
(4) $\phi\left(q, T_{r}^{f} x\right)+\phi\left(T_{r}^{f} x, x\right) \leq \phi(q, x)$ for all $x \in E$ and $q \in E P(f)$, which shows that $T_{r}^{f}$ is a quasi$\phi$-nonexpansive mapping

Now, we show that the mapping $I-T_{r}^{f}$ is demiclosedness at zero on a bounded subset of $E$.

Lemma 14. Let $E$ be a strictly convex, reflexive, and uniform smooth Banach space and $f: E \times E \longrightarrow \mathbb{R}$ be a bifunction satisfying conditions (A1)-(A4). Let $r>0$ and define the mapping $T_{r}^{f}$ as (86). Assume that $E P(f) \neq \varnothing$. Then, $I-T_{r}^{f}$ is demi-closedness at zero on a bounded set. That is, if $\left\{x_{n}\right\} \subset E$ is bounded and weakly converges to $x \in E$ and $\left\|x_{n}-\mathrm{T}_{r}^{f} x_{n}\right\| \longrightarrow 0$ as $n \longrightarrow \infty$, then $x=T_{r}^{f} x$.

Proof. et $\left\{x_{n}\right\} \subset E$ be bounded and converges weakly to $x \in E$ and $\left\|x_{n}-T_{r}^{f} x_{n}\right\| \longrightarrow 0$ as $n \longrightarrow \infty$. For each $x^{*} \in \operatorname{EP}(f)=\operatorname{Fix}\left(T_{r}^{f}\right)$, since $T_{r}^{f}$ is quasi- $\phi$-nonexpansive, we have

$$
\phi\left(x^{*}, T_{r}^{f} x_{n}\right) \leq \phi\left(x^{*}, x_{n}\right), \quad n \geq 1,
$$

which implies that $\left\{T_{r}^{f} x_{n}\right\}$ is bounded. On the contrary, since $J$ is uniformly norm-to-norm continuous on bounded sets, it follows that

$$
\lim _{n \longrightarrow \infty}\left\|J T_{r}^{f} x_{n}-J x_{n}\right\|=0 .
$$

By (A2), we have

$$
\begin{aligned}
\frac{1}{r}\left\langle y-T_{r}^{f} x_{n}, J T_{r}^{f} x_{n}-J x_{n}\right\rangle & \geq-f\left(T_{r}^{f} x_{n}, y\right) \\
& \geq f\left(y, T_{r}^{f} x_{n}\right), \quad \forall y \in E .
\end{aligned}
$$

Letting $n \longrightarrow 0$ in (89), by (A4) and (88), we obtain

$$
f(y, x) \leq 0, \quad \forall y \in E .
$$

For $0<t \leq 1$ and $y \in E$, let $y_{t}=t y+(1-t) x$. Note that (90) implies that $f\left(y_{t}, x\right) \leq 0$. By (A1), we have

$$
0=f\left(y_{t}, y_{t}\right) \leq t f\left(y_{t}, y\right)+(1-t) f\left(y_{t}, x\right) \leq t f\left(y_{t}, y\right) .
$$

Dividing by $t$, we obtain

$$
f\left(y_{t}, y\right) \geq 0, \quad \forall y \in E .
$$

Let $t \longrightarrow 0^{+}$, by (A3), we have

$$
f(x, y) \geq 0, \quad \forall y \in E .
$$

It follows that $x \in \operatorname{EP}(f)$. That is, $x=T_{r}^{f} x$ by Lemma 13 . This completes the proof.

Based on the results in Section 3, we give the following conclusion directly.

Theorem 3. Let $E_{1}$ be a 2-uniformly convex and 2-uniformly smooth real Banach space with the best smoothness constant $k>0$ and $E_{2}$ be a uniformly smooth, strictly convex, and reflexive Banach space. Let $A: E_{1} \longrightarrow E_{2}$ be a linear bounded operator with adjoint $A^{*}$. Let $f_{1}: E_{1} \times E_{1} \longrightarrow \mathbb{R}$ and $f_{2}: E_{2} \times E_{2} \longrightarrow \mathbb{R}$ be the bifunctions satisfying conditions (A1)-(A4). Assume that $\Lambda \neq \varnothing, \quad$ where $\Lambda=\left\{x \in E_{1}: x \in E P\left(f_{1}\right), A x \in E P\left(f_{2}\right)\right\}$. Let $r>0$. Take $x_{1} \in E_{1}$ and put $Q_{1}=E_{2}$. Define a sequence $\left\{x_{n}\right\}$ by

$$
\left\{\begin{array}{l}
w_{n}=T_{r}^{f_{2}} A x_{n}, \\
Q_{n}=\left\{w \in Q_{n}: \phi\left(w, w_{n}\right) \leq \phi\left(w, A x_{n}\right)\right\}, \\
z_{n}=J^{-1}\left(J_{1} x_{n}+\gamma_{n} A^{*} J_{2}\left(P_{Q_{n}}-I\right) A x_{n}\right), \\
y_{n}=J^{-1}\left[\left(1-\beta_{n}\right) J_{1} z_{n}+\left(1-\beta_{n}\right) J_{1} T_{r}^{f_{1}} z_{n}\right], \\
x_{n+1}=J_{1}^{-1}\left(\alpha_{n}\left(1-\tau_{n}\right) J_{1} x_{n}+\left(1-\alpha_{n}\right) J_{1} y_{n}\right), \quad n \geq 1,
\end{array}\right.
$$

where $\left\{\alpha_{n}\right\},\left\{\beta_{n}\right\} \subset(0,1),\left\{\tau_{n}\right\} \subset(\tau, 1)$ with $\tau \in(0,1)$ and

$$
\gamma_{n}= \begin{cases}\frac{\left\|\left(P_{Q_{n}}-I\right) A x_{n}\right\|^{2}}{2 k^{2}\left\|A^{*} J_{2}\left(I-P_{Q_{n}}\right) A x_{n}\right\|^{2}}, & \left\|\left(P_{Q_{n}}-I\right) A x_{n}\right\| \neq 0, \\ 0, & \text { else. }\end{cases}
$$

If the following conditions hold

$$
\begin{aligned}
\lim _{n \longrightarrow \infty} \alpha_{n} & =0, \\
\sum_{n=1}^{\infty} \alpha_{n} & =\infty \text { and } \liminf _{n \longrightarrow \infty} \beta_{\mathrm{n}}\left(1-\beta_{\mathrm{n}}\right)>0,
\end{aligned}
$$

then the sequence $\left\{x_{n}\right\}$ generated by (94) converges strongly to the element $x^{*}=\Pi_{\Lambda} \theta$, where $\theta$ is the zero element in $E_{1}$.

Theorem 4. Let $E_{1}$ be a 2-uniformly convex and 2-uniformly smooth real Banach space with the best smoothness constant $k>0$ and $E_{2}$ be a uniformly smooth, strictly convex, and reflexive Banach space. Let $A: E_{1} \longrightarrow E_{2}$ be a linear bounded operator with adjoint $A^{*}$. Let $f_{1}: E_{1} \times E_{1} \longrightarrow \mathbb{R}$ and $f_{2}: E_{2} \times E_{2} \longrightarrow \mathbb{R}$ be the bifunctions satisfying conditions (A1)-(A4). Assume that the interior of $\Lambda$ is nonempty, where $\Lambda=\left\{x \in E_{1}: x \in E P\left(f_{1}\right), A x \in E P\left(f_{2}\right)\right\}$. Let $r>0$. Take $u, x_{1} \in E_{1}$ and put $Q_{1}=E_{2}$. Define a sequence $\left\{x_{n}\right\}$ by

$$
\left\{\begin{array}{l}
w_{n}=T_{r}^{f_{2}} A x_{n}, \\
Q_{n}=\left\{w \in Q_{n}: \phi\left(w, w_{n}\right) \leq \phi\left(w, A x_{n}\right)\right\}, \\
z_{n}=J^{-1}\left(J_{1} x_{n}+\gamma_{n} A^{*} J_{2}\left(P_{Q_{n}}-I\right) A x_{n}\right), \\
y_{n}=J^{-1}\left[\left(1-\beta_{n}\right) J_{1} z_{n}+\left(1-\beta_{n}\right) J_{1} T_{r}^{f_{1}} z_{n}\right], \\
x_{n+1}=J_{1}^{-1}\left(\alpha_{n} J_{1} u+\left(1-\alpha_{n}\right) J_{1} y_{n}\right), \quad n \geq 1,
\end{array}\right.
$$

where $\left\{\alpha_{n}\right\},\left\{\beta_{n}\right\} \subset(0,1)$ and 


$$
\gamma_{n}= \begin{cases}\frac{\left\|\left(P_{Q_{n}}-I\right) A x_{n}\right\|^{2}}{2 k^{2}\left\|A^{*} J_{2}\left(I-P_{Q_{n}}\right) A x_{n}\right\|^{2}}, & \left\|\left(P_{Q_{n}}-I\right) A x_{n}\right\| \neq 0, \\ 0, & \text { else. }\end{cases}
$$

If the following conditions hold

$$
\begin{aligned}
& \lim _{n \longrightarrow \infty} \alpha_{n}=0, \\
& \sum_{n=1}^{\infty} \alpha_{n}<\infty \text { and } \liminf _{n \longrightarrow \infty} \beta_{\mathrm{n}}\left(1-\beta_{\mathrm{n}}\right)>0,
\end{aligned}
$$

then the sequence $\left\{x_{n}\right\}$ generated by (97) converges strongly to the element $x^{*}=\lim _{n \longrightarrow \infty} \Pi_{\Lambda} x_{n}$.

\section{Numerical Examples}

In this section, we give the following examples to illustrate the effectiveness of Algorithms 1 and 2. The program is performed by Matlab R2016b running on a PC Desktop with Core(TM) i5CPU M550 3.20 GHz with 4 GB Ram.

We first show the convergence of Algorithm 1 by the following example which has been used by Ma et al. [22]. In [22], the authors compare the computed results using their algorithm (25) with algorithm (100) in Kraikaew and Saejung [10] by the example. Here, we also compare the convergence of our Algorithm 1 with algorithm (25) in [22] and algorithm (100) in [10].

Example 1. Let $E_{1}=\mathbb{R}, E_{2}=\mathbb{R}^{2}, Q=[0, \infty] \times(-\infty, 0)$, $S x=(x / 4)$, for all $x \in E_{1}, T x=P_{Q} x$ for all $x \in E_{2}$, where $P_{Q}$ is the metric projection from $E_{2}$ onto $Q$, and $A: E_{1} \longrightarrow E_{2}$ be a mapping defined by $A x=(x / 2, x / 3)$ for all $x \in E_{1}$. Then, $A^{*}(u, v)=(u / 2)+(v / 3)$, for all $(u, v) \in E_{2}$. It is easy to see that $\Omega=\left\{x \in E_{1}: x \in \operatorname{Fix}(S), A x \in \operatorname{Fix}(T)\right\}=\{0\}$.

Algorithm 3. Let $\left\{x_{n}\right\}$ be the sequence generated by (25) in this paper with $\alpha_{n}=1 / 2 n$ and $\beta_{n}=\tau_{n}=6 / 7$. Then, scheme (25) can be simplified as

$$
\left\{\begin{array}{l}
x_{1} \in E_{1}, \\
w_{n}=P_{Q}\left(\frac{x_{n}}{2}, \frac{x_{n}}{3}\right) \\
Q_{n}=\left\{w \in E_{2}:\left\|w_{n}-w\right\| \leq\left\|\left(\frac{x_{n}}{2}, \frac{x_{n}}{3}\right)-w\right\|\right\} \\
A x_{n}=\left(\frac{x_{n}}{2}, \frac{x_{n}}{3}\right), z_{n}=x_{n}+\gamma_{n} A^{*}\left(P_{Q_{n}}-I\right) A x_{n} \\
y_{n}=\frac{6}{7} z_{n}+\frac{1}{28} z_{n}, \\
x_{n+1}=\frac{1}{14 n} x_{n}+\frac{2 n-1}{2 n} y_{n}, \quad n \geq 1
\end{array}\right.
$$

where

$$
\gamma_{n}= \begin{cases}\frac{\left\|\left(P_{Q_{n}}-I\right) A x_{n}\right\|^{2}}{2\left\|A^{*}\left(I-P_{Q_{n}}\right) A x_{n}\right\|^{2}}, & \text { if }\left\|\left(P_{Q_{n}}-I\right) A x_{n}\right\| \neq 0, \\ 0, & \text { otherwise. }\end{cases}
$$

Algorithm 4. Let $\left\{x_{n}\right\}$ be the sequence generated by algorithm (100) in [10] with $\alpha_{n}=1 / 2 n$ and $\gamma=1$. Then, scheme (100) in [10] can be simplified as

$$
x_{1} \in E_{1}, x_{n+1}=\frac{1}{2 n} x_{1}+\frac{2 n-1}{8 n}\left(x_{n}+A^{*}(T-I) A x_{n}\right), \quad n \geq 1 .
$$




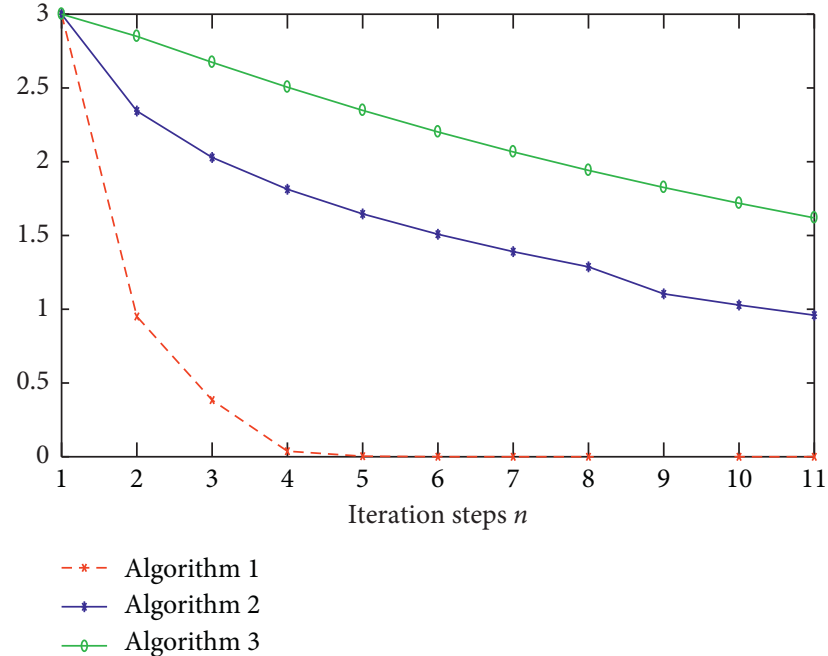

Figure 1: Convergence for Algorithms 3-5 with different initial points $x_{1}=3$.

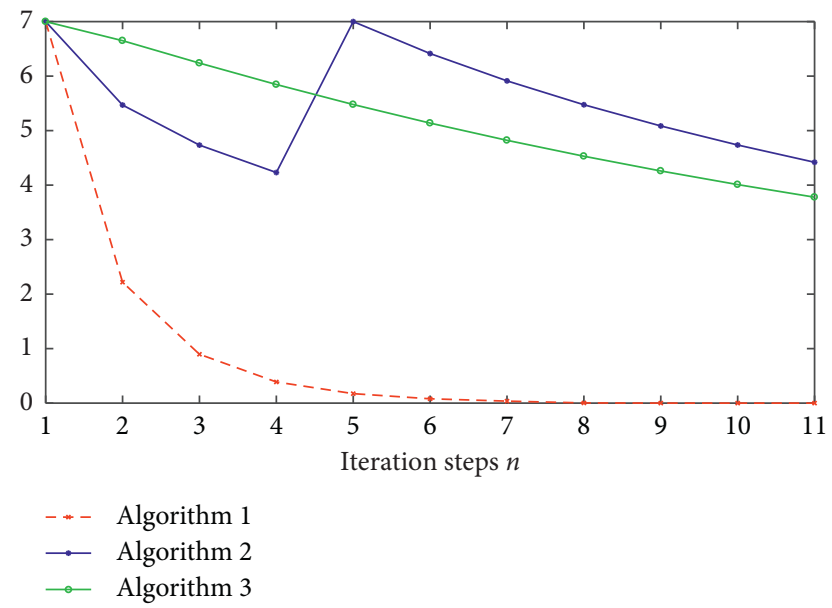

Figure 2: Convergence for Algorithms 3-5 with different initial points $x_{1}=7$.

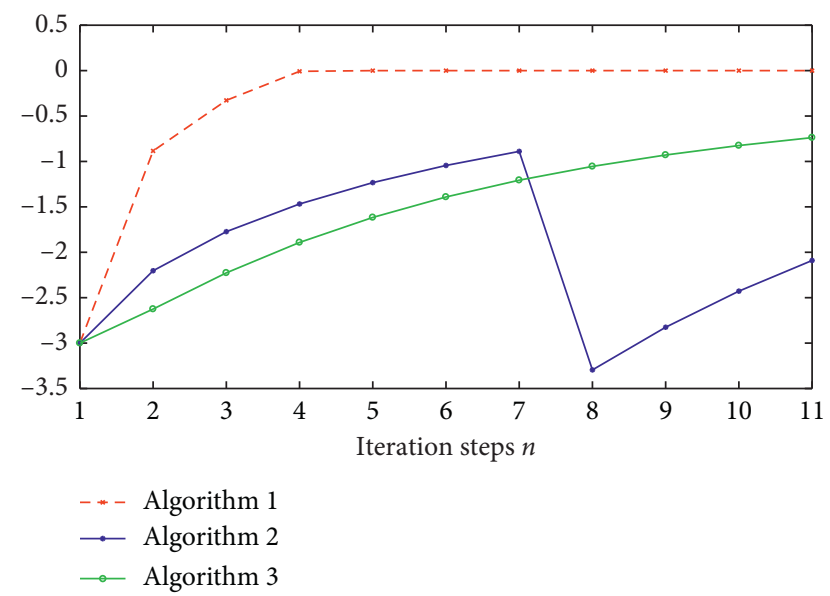

Figure 3: Convergence for Algorithms 3-5 with different initial points $x_{1}=-3$.

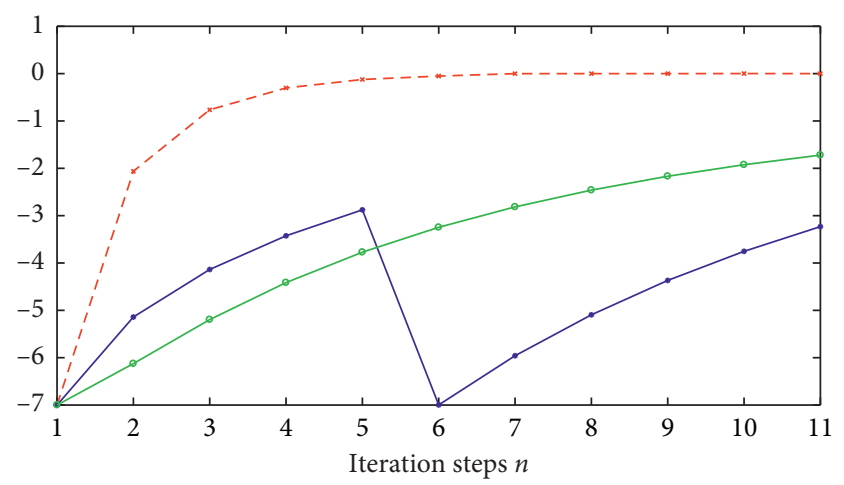

$$
\begin{array}{r}
\text {-. Algorithm 1 } \\
\text { - Algorithm 2 } \\
\text { - Algorithm } 3
\end{array}
$$

FIgURE 4: Convergence for Algorithms 3-5 with different initial points $x_{1}=-7$.

Algorithm 5. Let $\left\{x_{n}\right\}$ be the sequence generated by algorithm (25) in [22] with $\alpha_{n}=1 / 2 n$ and $\gamma=1$. Then, scheme (25) in [22] can be simplified as

$$
\left\{\begin{array}{l}
x_{1} \in E_{1}, \\
A x_{n}=\left(\frac{x_{n}}{2}, \frac{x_{n}}{3}\right), \\
z_{n}=x_{n}+A^{*}(T-I) A x_{n}, \\
y_{n}=\frac{2 n-1}{2 n} z_{n}+\frac{1}{8 n} z_{n}, \\
C_{n+1}=\left\{v_{n}: C_{n}:\left|y_{n}-v\right| \leq\left|x_{n}-v\right|,\left|z_{n}-v\right| \leq\left|x_{n}-v\right|\right\} \\
x_{n+1}=P_{C_{n+1}} x_{1}, \quad n \geq 1 .
\end{array}\right.
$$

We perform schemes (100)-(103) with the different initial points. Figures $1-4$ show that the sequence $\left\{x_{n}\right\}$ generated by (100)-(103) converge to 0 .

Remark 2. (a) Although Theorem 1 in [22] requires that $\left\{\alpha_{n}\right\}$ in Algorithm 5, i.e., algorithm (25) in [22], takes values in $[\delta, 1)$ with $\delta \in(0,1)$; here, for comparing the convergence rate of three schemes, we put the same $\alpha_{n}=1 / 2 n$. This does not affect the effectiveness of Algorithm 5 since the program stops in finite iterations. (b) Figures 1-4 above show that the convergence rate of Algorithm 3 is faster than that of Algorithms 4 and 5 .

Next, we illustrate Theorem 2 by the following example.

Example 2. Let $E_{1}=\mathbb{R}^{2}$ and $E_{2}=\mathbb{R}$. Define the mappings $S: E_{1} \longrightarrow E_{1}$ by $S x=\left(\left(x_{1} / 2\right), x_{2}\right)$ for all $x=\left(x_{1}, x_{2}\right) \in E_{1}$, and $T: E_{2} \longrightarrow E_{2}$ by $T x=x / 2$ if $|x| \leq 1$ and $T x=1$ if $|x|>1$. 
TABLe 1: Convergence for Algorithm 2 with initial point $x_{1}=(3,6)$.

\begin{tabular}{lc}
\hline Iteration steps & $x_{n}$ \\
\hline 1 & $(-2,-5)$ \\
2 & $(-1.48705,-5.00000)$ \\
3 & $(-1.13985,-4.99999)$ \\
4 & $(-0.89031,-4.99999)$ \\
5 & $(-0.70347,-4.99999)$ \\
$\vdots$ & $\vdots$ \\
100 & $(-0.00085,-4.99999)$ \\
261 & $(-0.00012,-4.99998)$ \\
262 & $(-0.00012,-4.99998)$ \\
263 & $(-0.00011,-4.99998)$ \\
264 & $(-0.00011,-4.99998)$ \\
265 & $(-0.00011,-4.99998)$ \\
$\vdots$ & $\vdots$ \\
286 & $(-0.00009,-4.99998)$ \\
\hline
\end{tabular}

TABLE 2: Convergence for Algorithm 2 with initial point $x_{1}=(3,6)$.

\begin{tabular}{lc}
\hline Iteration steps & $x_{n}$ \\
\hline 1 & $(3,6)$ \\
2 & $(2.23056,0.99999)$ \\
3 & $(1.70977,1.55555)$ \\
4 & $(1.33545,1.83331)$ \\
$\vdots$ & $\vdots$ \\
100 & $(0.00128,1.99985)$ \\
101 & $(0.00125,2.00024)$ \\
102 & $(0.00122,2.00022)$ \\
300 & $(0.00014,1.99997)$ \\
301 & $(0.00013,2.00002)$ \\
302 & $(0.00013,2.00003)$ \\
303 & $(0.00013,1.99997)$ \\
$\vdots$ & $\vdots$ \\
349 & $(0.00010,2.00002)$ \\
350 & $(0.00010,2.00000)$ \\
351 & $(0.00009,2.00003)$ \\
\hline
\end{tabular}

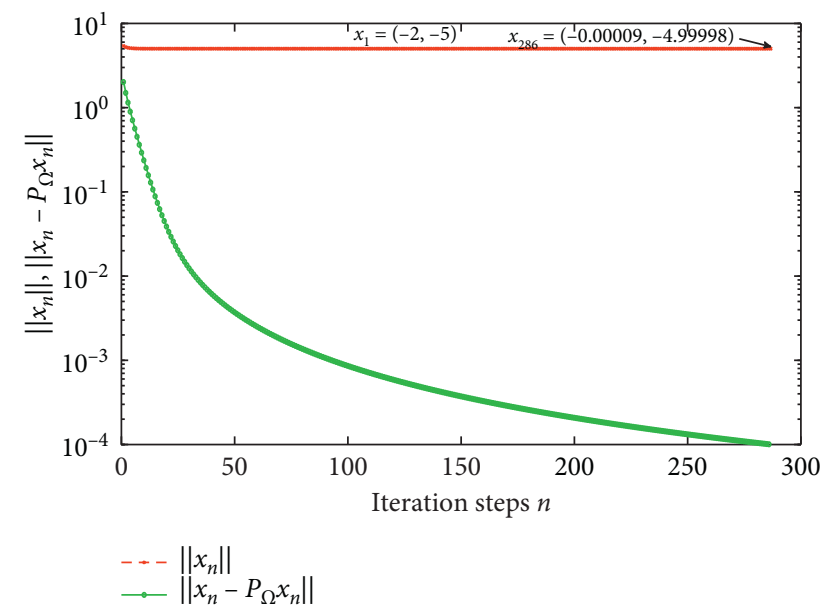

FIgURe 5: Convergence for Algorithm 2 with different initial points $x_{1}=(-2,-2)$

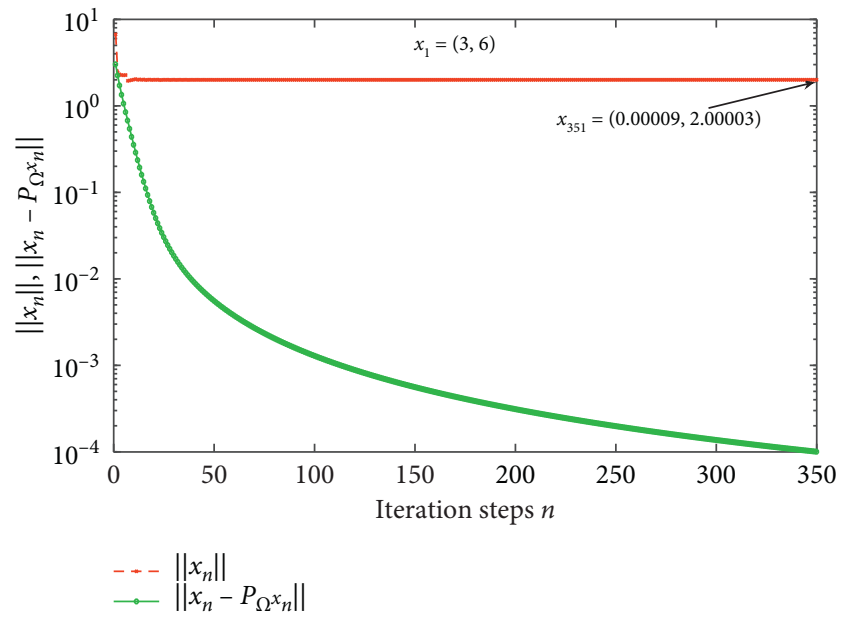

FIgURE 6: Convergence for Algorithm 2 with different initial points $x_{1}=(3,6)$.

Let $A: E_{1} \longrightarrow E_{2}$ be a mapping defined by $A x=x_{1}$ for all $x=\left(x_{1}, x_{2}\right) \in E_{1}$. Then, $A$ is linear and bounded and $A^{*} y=(y, 0)$ for all $y \in E_{2}$. It is easy to see that $\Omega=\left\{\left(0, x_{2}\right): x_{2} \in \mathbb{R}\right\}$. All the conditions on $S, T$, and $\Omega$ are satisfied for Theorem 2 .

By Algorithm 2, we generate a sequence $\left\{x_{n}\right\}$ with $\alpha_{n}=$ $1 / n^{2}$ and $\beta_{n}=1 / 2\left(1-e^{(n / 2)}\right)$ for all $n \geq 1$. Theorem 2 shows that $\left\{x_{n}\right\}$ will converge to the point $P_{\Omega} x_{n}$. We will stop the program when $\left\|x_{n}-P_{\Omega} x_{n}\right\|<10^{-4}$. The computed results of the sequence $\left\{x_{n}\right\}$ are given in Tables 1 and 2 . Figures 5 and 6 show the convergence of the sequence $\left\{x_{n}\right\}$.

\section{Conclusion}

For finding a solution of the split common fixed problem of quasi- $\phi$-nonexpansive mappings in Banach space, we introduced a Halpern algorithm and a nonconvex combination algorithm where the norm of the linear bounded operator does not need to be known in advance. The convergence of the algorithms was investigated and some numerical examples were given to illustrate the convergence of the algorithms.

\section{Data Availability}

All data for our algorithms are included in this paper.

\section{Conflicts of Interest}

The author declares that there are no conflicts of interest.

\section{References}

[1] Y. Censor and T. Elfving, "A multiprojection algorithm using Bregman projections in a product space," Numerical Algorithms, vol. 8, no. 2, pp. 221-239, 1994.

[2] N. T. An, N. M. Nam, and X. Qin, "Solving $k$-center problems involving sets based on optimization techniques," Journal of Global Optimization, vol. 76, no. 1, pp. 189-209, 2020.

[3] Y. Censor, T. Bortfeld, B. Martin, and A. Trofimov, "A unified approach for inversion problems in intensity modulated 
radiation therapy," Physics in Medicine and Biology, vol. 51, pp. 2356-2365, 2006.

[4] T. H. Cuong, J.-C. Yao, and N. D. Yen, "Qualitative properties of the minimum sum-of-squares clustering problem," Optimization, vol. 69, no. 9, pp. 2131-2154, 2020.

[5] L. V. Nguyen and X. Qin, "The minimal time function associated with a collection of sets," ESAIM: Control, Optimisation and Calculus of Variations, vol. 26, p. 93, 2020.

[6] C. Byrne, "Iterative oblique projection onto convex sets and the split feasibility problem," Inverse Problems, vol. 18, no. 2, pp. 441-453, 2002.

[7] C. Byrne, "A unified treatment of some iterative algorithms in signal processing and image reconstruction," Inverse Problems, vol. 20, no. 1, pp. 103-120, 2004.

[8] H.-K. Xu, "Iterative methods for the split feasibility problem in infinite-dimensional Hilbert spaces," Inverse Problems, vol. 26, no. 10, Article ID 105018, 2010.

[9] A. Moudafi, "The split common fixed point problem for demicontractive mapping," Inverse Problems, vol. 26, Article ID 055007, 2010.

[10] R. Kraikaew and S. Saejung, "On split common fixed point problems," Journal of Mathematical Analysis and Applications, vol. 415, no. 2, pp. 513-524, 2014.

[11] A. Moudafi, "A note on the split common fixed-point problem for quasi-nonexpansive operators," Nonlinear Analysis: Theory, Methods \& Applications, vol. 74, no. 12, pp. 4083-4087, 2011.

[12] A. Moudafi, “A relaxed alternating CQ-algorithm for convex feasibility problems," Nonlinear Analysis: Theory, Methods \& Applications, vol. 79, pp. 117-121, 2013.

[13] X. L. Qin, L. Wang, and J. C. Yao, "Inertial splitting method for maximal monotone mappings," Journal of Nonlinear Convex Analysis, vol. 21, pp. 2325-2333, 2020.

[14] W. Takahashi, C. F. Wen, and J. C. Yao, "The shrinking projection method for a finite family of demimetric mappings with variational inequality problems in a Hilbert space," Fixed Point Theory, vol. 19, pp. 407-419, 2018.

[15] W. Takahahsi and J. C. Yao, "The split common fixed point problem for two finite families of nonlinear mappings in Hilbert spaces," Journal of Nonlinear Convex Analysis, vol. 20, pp. 173-195, 2019.

[16] F. Schopfer, T. Schuster, and A. K. Louis, "An iterative regularization method for the solution of the split feasibility problem in Banach spaces," Inverse Problems, vol. 24, Article ID 055008, 20 pages, 2008.

[17] J. F. Tang, S. S. Chang, L. Wang, and X. R. Wang, "On the split common fixed point problem for strict pseudocontractive and asymptotically nonexpansive mappings in Banach spaces," Journal Inequalities Application, vol. 305, p. 2015, 2015.

[18] J.-Z. Chen, H.-Y. Hu, and L.-C. Ceng, "Strong convergence of hybrid Bregman projection algorithm for split feasibility and fixed point problems in Banach spaces," The Journal of Nonlinear Sciences and Applications, vol. 10, no. 1, pp. 192204, 2017.

[19] S. Y. Cho, "Implicit extragradient-like method for fixed point problems and variational inclusion problems in a Banach space," Symmetry, vol. 12, no. 6, p. 998, 2020.

[20] Z. Ma and L. Wang, "On the split equality common fixed point problem for asymptotically nonexpansive semigroups in Banach spaces," Journal of Nonlinear Sciences and Applications, vol. 09, no. 6, pp. 4003-4015, 2016.

[21] X. Tian, L. Wang, and Z. Ma, "On the split equality common fixed point problem for quasi-nonexpansive multi-valued mappings in Banach spaces," Journal of Nonlinear Sciences and Applications, vol. 9, no. 10, pp. 5536-5543, 2016.

[22] Z. Ma, L. Wang, and S.-S. Chang, "On the split feasibility problem and fixed point problem of quasi- $\phi$-nonexpansive mapping in banach spaces," Numerical Algorithms, vol. 80, no. 4, pp. 1203-1218, 2019.

[23] F. Kohasaka and W. Takahashi, "Strong convergence of an iterative sequence for maximal monotone operators in Banach spaces," Abstract and Applied Analysis, vol. 3, pp. 239-249, 2004.

[24] X. Qin, Y. J. Cho, and S. M. Kang, "Convergence theorems of common elements for equilibrium problems and fixed point problems in Banach spaces," Journal of Computational and Applied Mathematics, vol. 225, no. 1, pp. 20-30, 2009.

[25] H. Zegeye, "A hybrid iteration scheme for equilibrium problems, variational inequality problems and common fixed point problems in banach spaces," Nonlinear Analysis: Theory, Methods \& Applications, vol. 72, no. 3-4, pp. 2136-2146, 2010.

[26] D. V. Hieu and J. J. Strodiot, "Strong convergence theorems for equilibrium problems and fixed point problems in Banach spaces," Journal of Fixed Point Theory and Applications, vol. 20, no. 20, p. 131, 2018.

[27] N. Hussain, G. Marino, L. Muglia, and B. A. Alamri, "On some Mann's type iterative algorithms," Fixed Point Theory Application, vol. 17, 2015.

[28] G. Marino, B. Scardamaglia, and E. Karapinar, "Strong convergence theorem for strict pseudo-contractions in Hilbert spaces," Journal of Inequalities Application, vol. 24, p. 134, 2016.

[29] S. Kamimura and W. Takahashi, "Strong convergence of a proximal-type Algorithm in a Banach space," SIAM Journal on Optimization, vol. 13, no. 3, pp. 938-945, 2002.

[30] Y. I. Alber and S. Reich, "An iterative method for solving a class of nonlinear operator equations in Banach spaces," Panamerican Mathematical Journal, vol. 4, pp. 39-54, 1994.

[31] H. Y. Zhou and X. L. Qin, Fixed Points of Nonlinear Operators. Iterative Methods, De Gruyter, Berlin, Germany, 2020.

[32] Y. I. Alber, "Metric and generalized projection operators in Banach spaces: properties and applications, in theory and applications of nonlinear operators of accretive and monotone type," in Lecture Notes in Pure and Applied Mathematics, Dekker, New York, NY, USA, 1996.

[33] H.-K. Xu, "Inequalities in banach spaces with applications," Nonlinear Analysis: Theory, Methods \& Applications, vol. 16, no. 12, pp. 1127-1138, 1991.

[34] H.-K. Xu, "Another control condition in an iterative method for nonexpansive mappings," Bulletin of the Australian Mathematical Society, vol. 65, no. 1, pp. 109-113, 2002.

[35] P.-E. Maingé, "Strong convergence of projected subgradient methods for nonsmooth and nonstrictly convex minimization," Set-Valued Analysis, vol. 16, no. 7-8, pp. 899-912, 2008.

[36] K. K. Tan and H. K. Xu, "Approximating fixed points of nonexpansive mappings by the Ishikawa iteration process," Journal of Mathematical Analysis and Applications, vol. 178, no. 2, pp. 301-308, 1993.

[37] S. Y. Cho and X. Qin, "On the strong convergence of an iterative process for asymptotically strict pseudocontractions and equilibrium problems," Applied Mathematics and Computation, vol. 235, pp. 430-438, 2014.

[38] S. Y. Cho, "A convergence theorem for generalized mixed equilibrium problems and multivalued asymptotically nonexpansive mappings," Journal of nonlinear and convex analysis, vol. 21, pp. 1017-1026, 2020. 
[39] Z. H. He, "The split equilibrium problem and its convergence algorithms," Journal of Inequalities and Applications, vol. 2012, p. 162, 2012.

[40] K. R. Kazmi and S. H. Rizvi, "Iterative approximation of a common solution of a split equilibrium problem, a variational inequality problem and a fixed point problem," Journal of the Egyptian Mathematical Society, vol. 21, no. 1, pp. 44-51, 2013.

[41] L. V. Nguyen, Q. H. Ansari, and X. L. Qin, "Linear conditioning, weak sharpness and finite convergence for equilibrium problems," Journal of Global Optimization, vol. 77, pp. $405-424,2020$. 